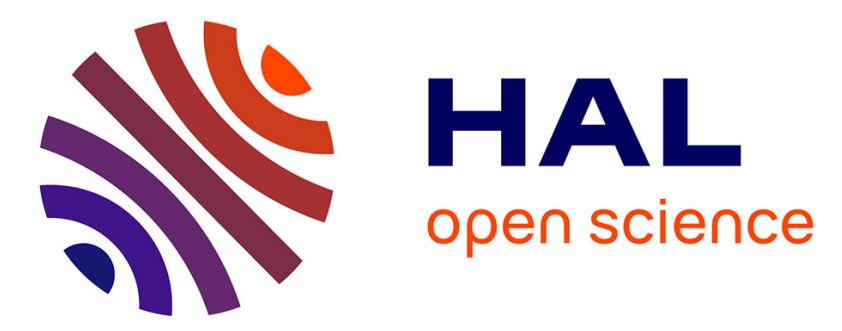

\title{
Optically anisotropic media: New approaches to the dielectric function, singular axes, microcavity modes and Raman scattering intensities
}

Marius Grundmann, Chris Sturm, Christian Kranert, Steffen Richter, Rüdiger Schmidt-Grund, Jesús Zúñiga-Pérez, Christiane Deparis

\section{To cite this version:}

Marius Grundmann, Chris Sturm, Christian Kranert, Steffen Richter, Rüdiger Schmidt-Grund, et al.. Optically anisotropic media: New approaches to the dielectric function, singular axes, microcavity modes and Raman scattering intensities. physica status solidi (RRL) - Rapid Research Letters, 2016, 11 (1), pp.1600295. 10.1002/pssr.201600295 . hal-03484138

\section{HAL Id: hal-03484138 \\ https://hal.science/hal-03484138}

Submitted on 10 Jan 2022

HAL is a multi-disciplinary open access archive for the deposit and dissemination of scientific research documents, whether they are published or not. The documents may come from teaching and research institutions in France or abroad, or from public or private research centers.
L'archive ouverte pluridisciplinaire HAL, est destinée au dépôt et à la diffusion de documents scientifiques de niveau recherche, publiés ou non, émanant des établissements d'enseignement et de recherche français ou étrangers, des laboratoires publics ou privés. 


\title{
Optically Anisotropic Media:
} New Approaches to the Dielectric

Function, singular axes, Microcavity

Modes and Raman Scattering

\section{Intensities}

\author{
Marius Grundmann ${ }^{*}, 1$, Chris Sturm ${ }^{1}$, Christian Kranert ${ }^{1}$, Steffen Richter ${ }^{1}$, Rüdiger Schmidt-Grund ${ }^{1}$, \\ Christianne Deparis ${ }^{2}$, Jesús Zúñiga-Pérez ${ }^{2}$ \\ ${ }^{1}$ Institut für Experimentelle Physik II, Universität Leipzig, Linnéstr. 5, 04103 Leipzig, Germany \\ ${ }^{2}$ CRHEA-CNRS, Rue Bernard Grégory, 06560 Valbonne, France
}

Received XXXX, revised XXXX, accepted XXXX

Published online XXXX

Key words: Anisotropic optical media, birefringence, Raman scattering, microcavities, dielectric function, exceptional points, GaN, $\mathrm{ZnO}, \mathrm{Ga}_{2} \mathrm{O}_{3}$.

* Corresponding author: e-mail grundmann@physik.uni-leipzig.de, Phone: +49-341-9732650, Fax: +49-341-9732668

Six out of seven crystal systems are optically anisotropic and birefringent. We review recent insight that biaxial crystals generally exhibit four singular axes (or exceptional points) which can pairwise degenerate for special cases. Planar anisotropic microcavities are discussed as effectively biaxial systems and we predict exceptional points and demonstrate partially coalesced eigenstates. Also the general form of the dielectric function of anisotropic crystals based on individual dipole oscillators for phonon and electronic resonance is discussed. The impact of birefringence on Raman scattering intensities has been historically either ignored or modeled incorrectly. A recent theory for uniaxial and biaxial crystals explains experimental Raman scattering intensities for excitation off the principal directions without free parameters, allowing the unambiguous determination of the Raman tensor components. The above points are demonstrated and relevant in particular for the currently technologically important materials GaN, $\mathrm{ZnO}$ (uniaxial) and $\beta-\mathrm{Ga}_{2} \mathrm{O}_{3}$ (biaxial).

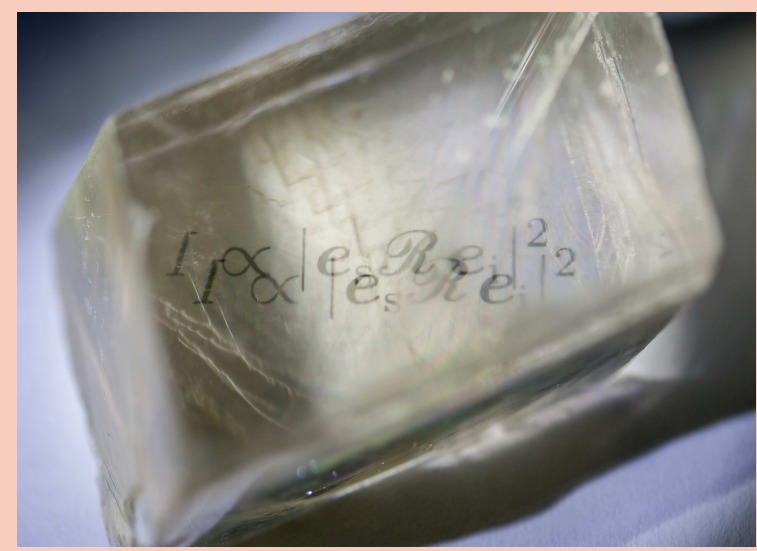

Formula for the Raman scattering intensity as a function of incoming and outgoing polarization and the Raman tensor seen through birefringent crystal (calcite).

Copyright line will be provided by the publisher

1 Introduction There exist seven crystal systems, cubic, tetragonal, hexagonal, trigonal, orthorhombic, monoclinic and triclinic. It is well known that the dielectric tensor for a suitable high symmetry choice of coordinate sys- tem has the forms given in Table 1; the optical character is denoted as isotropic, uniaxial (for tetragonal, hexagonal, trigonal crystals) or biaxial (for orthorhombic, monoclinic and triclinic crystals) [1]. Optically isotropic (cubic) crys- 
Table 1 General form of the dielectric tensor $\epsilon$ for the 7 crystal systems, maximum number of optic axes $N$ (for complex $\epsilon$, indices 's' and 'n' denote singular and normal optic axes) and number of different indices of refraction of these axes $\nu$.

\begin{tabular}{|c|c|c|c|c|c|}
\hline $\begin{array}{l}\text { crystal } \\
\text { system }\end{array}$ & $\begin{array}{l}\text { optical } \\
\text { symmetry }\end{array}$ & $\epsilon$ & examples & $N$ & $\nu$ \\
\hline cubic & isotropic & $\left(\begin{array}{lll}a & 0 & 0 \\
0 & a & 0 \\
0 & 0 & a\end{array}\right)$ & $\begin{array}{l}\mathrm{Si}, \mathrm{GaAs}, \\
\mathrm{MgO}, \mathrm{CuI}\end{array}$ & $\infty_{n}$ & 1 \\
\hline $\begin{array}{l}\text { tetragonal } \\
\text { hexagonal } \\
\text { trigonal }\end{array}$ & uniaxial & $\left(\begin{array}{lll}a & 0 & 0 \\
0 & a & 0 \\
0 & 0 & c\end{array}\right)$ & $\begin{array}{l}\mathrm{CuGaSe}_{2} \\
\mathrm{GaN}, \mathrm{ZnO} \\
\mathrm{Bi}_{2} \mathrm{Se}_{3}\end{array}$ & $1_{\mathrm{n}}$ & 1 \\
\hline orthorhombic & biaxial & $\left(\begin{array}{lll}a & 0 & 0 \\
0 & b & 0 \\
0 & 0 & c\end{array}\right)$ & $\begin{array}{l}\mathrm{Sb}_{2} \mathrm{Se}_{3}, \\
\mathrm{SrSO}_{4}\end{array}$ & $4_{\mathrm{s}}$ & 1 \\
\hline monoclinic & biaxial & $\left(\begin{array}{lll}a & 0 & d \\
0 & b & 0 \\
d & 0 & c\end{array}\right)$ & $\begin{array}{l}\beta-\mathrm{Ga}_{2} \mathrm{O}_{3}, \\
\text { anthracene }\end{array}$ & $4_{\mathrm{s}}$ & 2 \\
\hline triclinic & biaxial & $\left(\begin{array}{lll}a & d & e \\
d & b & f \\
e & f & c\end{array}\right)$ & $\begin{array}{l}\mathrm{K}_{2} \mathrm{Cr}_{2} \mathrm{O}_{7} \\
\text { tetracene }\end{array}$ & $4_{\mathrm{s}}$ & 4 \\
\hline
\end{tabular}

tals can also become optically anisotropic (uniaxial or biaxial) in the presence of non-hydrostatic strain or in the presence of (anisotropic) surface layers.

Here we review recent insights into the optical properties of anisotropic crystals. The general form of the dielectric tensor and its deconstruction into the dipole contributions of lattice and electronic oscillators oriented in different directions is discussed. Also we point out that in general biaxial materials possess four "singular" optic axes which can pairwise degenerate for special cases, in particular when the dielectric tensor is real (transparency regime). The presence of four "Singularitäts- oder Windungsaxen" in biaxial, absorbing crystals has been pointed out first by W. Voigt [2] (Fig. 1) in 1902 [8-10]. For these directions the eigenstate is left- or right-circular polarized and accordingly allows the propagation of so-called Voigt waves.

The case of transparent biaxial crystals is still very interesting [11], historically well-known for the effect of conical refraction $[12,13]$, an optical singularity [14] due to the double cone structure of the index of refraction around the optic axes. Recent experiments also discuss the spin and angular momentum control of beams using conical refraction [15]. Here, however, we focus on the regime where the imaginary part of the dielectric function cannot be neglected.

The discovery of singular axes was preceeded by Laspeyres who stated in 1880 that (in modern terms) the main axes of the real and imaginary parts of the dielectric tensor generally do not coincide $[16,17]$. Before that Babinet had worked on the relation of the direction of fastest wave and strongest absorption [18]. Voigt's work of 1884 [19] on weakly absorbing (biaxial) crystals is written in a pre-Maxwell formulation. For weak absorption it was stated that two optic axes exist with different attenuation of two elliptically polarized waves. The orientation of absorption axes and their symmetry for orthorhombic and monoclinic crystals was also discussed in 1887 by Becquerel [20]. The problem was then approached in the well known paper of Drude [21], summarizing his thesis as Voigt's doctoral student, on reflection and refraction at the interface of absorbing crystals. Here Drude develops a general treatment but states that optic axes do not exist in absorbing crystals of the orthorhombic system [22]. A summary of early crystal optics around this time can be found in [23].

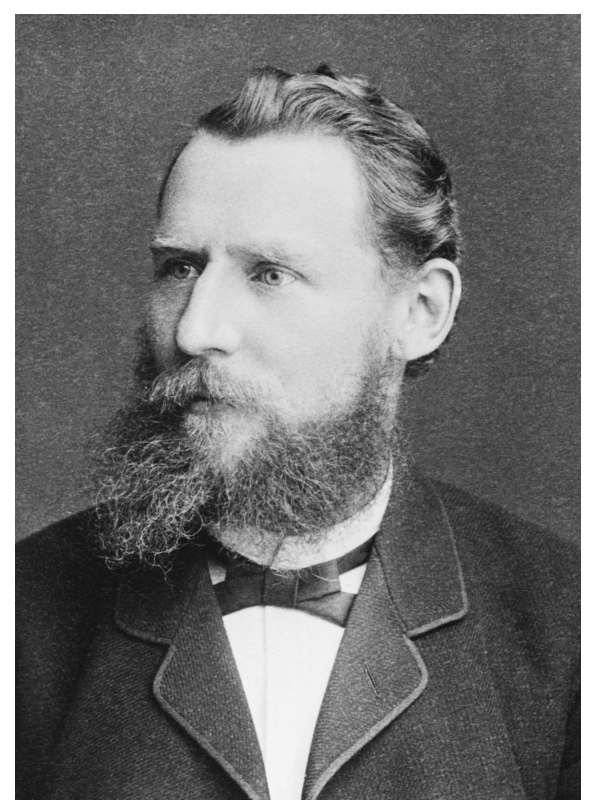

Figure 1 Woldemar Voigt (1850-1919) [24].

Finally in [8] Voigt uses the "Maxwell-Hertz" formulas and states that the main axes of the real and the imaginary parts are differently oriented in general, naming them polarization and absorption axes. The general case for a symmetric dielectric tensor and weak absorption is treated. Experimental observations on orthorhombic (andalusite), monoclinic (epidote) and triclinic (axinite) crystals are discussed and explained. He revisited this topic several times [25-29].

Further work on singular axes has been reported e.g. in [30-41], but we feel that parts of the optics community have largely forgotten about singular axes. A recent analysis provided an in depth view of the spectral dispersion and possible degeneracies of the singular axes [42] for monoclinic gallia as model system.

We note that in a more general framework such degeneracies of non-Hermitian Hamiltonians [43-46] with 
chiral and coalesced eigenfunctions are termed exceptional points [47-52] and have been found in coupled dissipative dynamical systems [53], mechanical problems [54], electronic circuits [55], microwave cavities [56], gyrokinetics of plasmas [57], coupled fiber-ring resonators [58], atomic spectra $[59,60]$, coupled quantum cascade microdisk lasers [61], photonic crystals [62], inhomogeneous gain media [63], optical lattices of driven cold atoms [64] and plasmonic waveguides [65]. We also point to literature on exceptional points with a degeneracy larger than two [62, 66-68].

The associated effects of birefringence in uniaxial and biaxial crystals affect Raman scattering for crystal orientations off the principal axes, a problem first posed by Michalke in 1938 [69]. After several works, the topic was dropped unresolved and Raman scattering intensities from optically anisotropic crystals not oriented along a principal direction where explained with the help of unphysical parameters, e.g. by including an ad hoc complex phase factor into the technically real Raman tensor (in the transparency regime) for hexagonal (and thus uniaxial) $\mathrm{GaN}$ [70] or $\mathrm{ZnO}$ [71] or several phase factors for orthorhombic (and thus biaxial) black phosphorus [72] or denoting the squares of Raman tensor elements for (monoclinic) naphthalene and anthracene [73]. Only recently, a theory was conceptualized to explain Raman scattering intensities for all crystal systems taking birefringence properly into account [74]; these results are for example highly relevant for the technologically interesting non-polar and semi-polar orientations of wurtzite materials [75].

From the materials $\mathrm{ZnO}$ and $\mathrm{GaN}$ microcavities have been built that enable strong light-matter coupling and condensation of exciton-polaritons at elevated temperature and thus macroscopic quantum states and new laser mechanisms up to room temperature. A particularly interesting case, related to the previously discussed biaxial crystal optics problems, are cavities for which the optic axis is tilted (typically perpendicular) to the cavity layer direction. Such "effectively biaxial" inhomogeneous media are discussed at the end of this review. We predict the occurrence of exceptional points and show first experimental evidence for non-orthogonal (partially coalesced, elliptically polarized) eigenstates in such cavity. We note that Voigt waves have been discussed in composite media from two uniaxial materials with relative tilt of their optic axes in [37].

The calculations for this paper have been executed using Mathematica [76], except the calculations for the planar microcavities which have been executed using MATLAB [77].

2 Dielectric Function Only for a few optically anisotropic materials the dielectric function has been reported in a wide wavelength range. For monoclinic gallia $\left(\beta-\mathrm{Ga}_{2} \mathrm{O}_{3}\right)$ the dielectric tensor has been determined using generalized spectroscopic ellipsometry in the energy range $0.5-$ $8.5 \mathrm{eV}$ [78] on crystals purchased from Tamura Corpora- tion [79]. An extension to the infrared phonon regime was reported in [80] down to about $0.03 \mathrm{eV}$ and in [81] down to about $0.006 \mathrm{eV}$. In $[82,83]$ a method in the infrared based on external and total internal reflection was reported in order to determine phonon oscillator parameters in monoclinic crystals from reflectivity spectra.

Extending an approach of [84-86] beyond Lorentz oscillators, the dielectric response is considered (in first order) as the combined effect of various spectrally broadened and spectrally overlapping oscillators with their dipoles oriented with respect to the coordinate system of the (relative) dielectric tensor (generally $\epsilon_{i j}=\delta_{i j}+\chi_{i j}$ ),

$$
\boldsymbol{\epsilon}=\mathbb{1}+\sum_{i=1}^{N} R\left(\phi_{i}, \theta_{i}\right) \chi_{i}^{\prime} R^{-1}\left(\phi_{i}, \theta_{i}\right),
$$

$\chi_{i}^{\prime}$ being the susceptibility (tensor) of the $i^{t h}$ excitation and $\phi_{i}$ and $\theta_{i}$ denoting the Euler angles, which are in general different for each excitation, and $R$ being the rotation matrix [80]. Eq. (1) is valid in general and necessary for a triclinic material. Allowed dipole orientations are restricted by crystal symmetry and allow to simplify (1) to

$$
\boldsymbol{\epsilon}=\mathbb{1}+\sum_{i=1}^{N_{y}} \chi_{i, y}+\sum_{j=1}^{N_{x z}} R\left(\phi_{j}\right) \chi_{j, x z}^{\prime} R^{-1}\left(\phi_{j}\right),
$$

for a monoclinic material (symmetry forcing dipoles to be polarized either along $y$ or perpendicular to $y$, in the $(x, z)$ plane), and to

$$
\boldsymbol{\epsilon}=\mathbb{1}+\sum_{i=1}^{N_{x}} \boldsymbol{\chi}_{i, x}+\sum_{j=1}^{N_{y}} \boldsymbol{\chi}_{j, y}+\sum_{k=1}^{N_{z}} \boldsymbol{\chi}_{k, z},
$$

for an orthorhombic material [80].

The validity of (2) for the application to $\beta-\mathrm{Ga}_{2} \mathrm{O}_{3}$ in the phonon range [80,81] and for electronic transitions [80] has been confirmed. In Fig. 2 the unit cell of $\beta-\mathrm{Ga}_{2} \mathrm{O}_{3}$ is shown with the $y$-direction perpendicular to the $(x, z)$ plane containing the non-rectangular angle.

The experimentally found dipole orientations (visualized in Fig. 3) coincide with theoretically calculated ones very closely and allow deeper insights in the atomistic origin of the various lattice and electronic contributions [80]. For the phonon modes also the (relative) oscillator strengths are well reproduced by theory. We note that a modified Lyddane-Sachs-Teller relation for monoclinic and triclinic materials has been proposed in [87].

In heteroepitaxy rotation domains can occur when the symmetries of substrate and epilayer do not match [88, 89]. The associated averaging of the dielectric tensor over various domain orientations can modify the effective optical properties. In the case of epitaxy of $\beta-\mathrm{Ga}_{2} \mathrm{O}_{3}$ on [00.1]-oriented (c-plane) $\mathrm{Al}_{2} \mathrm{O}_{3}$ six different (201)oriented domains occur [90] and the resulting effective medium (for measurement averaging over many domains) is uniaxial $[80,91]$. 


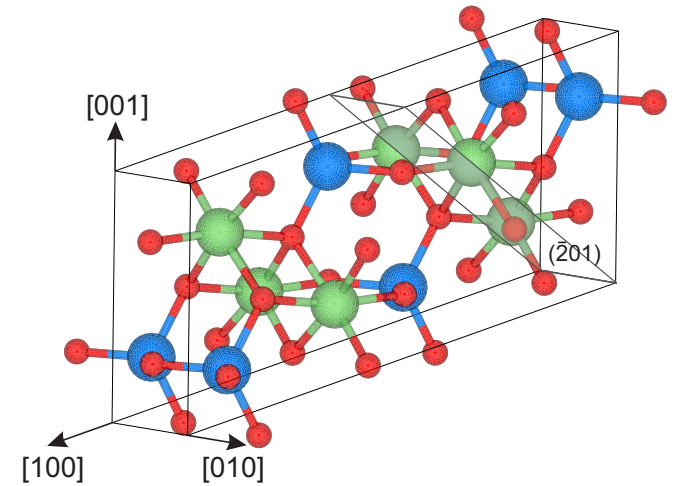

Figure 2 Unit cell of $\beta-\mathrm{Ga}_{2} \mathrm{O}_{3}$ (oxygen atoms are shown in red, gallium atoms as green (octahedral-like coordination) and blue (tetrahedral-like coordination)) and its orientation in the coordinate system chosen in Table 1: [100] $\| x$, [010] $\| y$; the angle between $z=x \times y$ and [001] is $103.7^{\circ}-\pi / 2$. The $(\overline{201})$ plane is indicated.
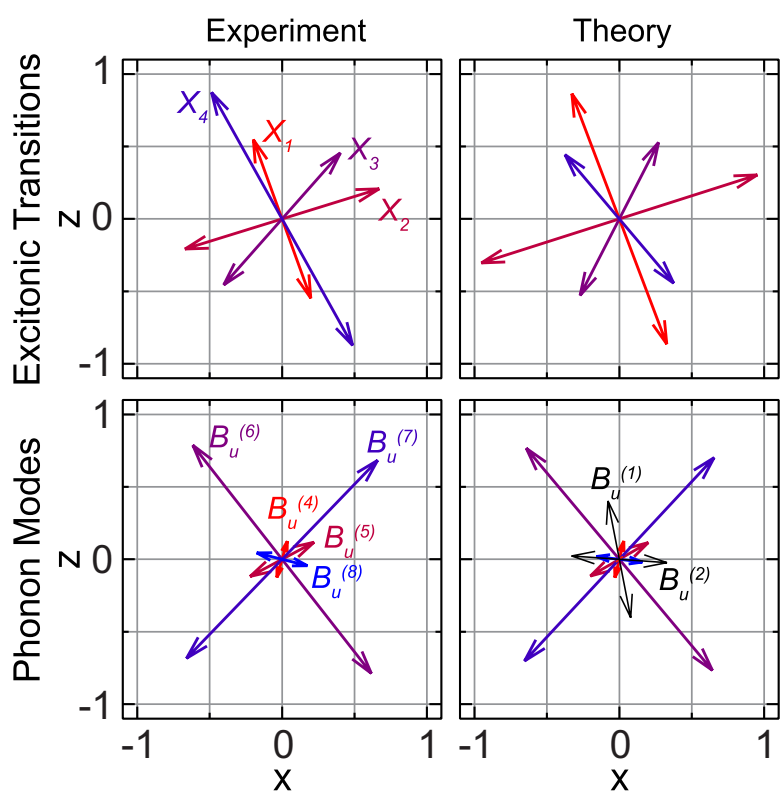

Figure 3 Arrows depicting the orientation and relative amplitudes (the strongest transitions is scaled to unit length) of the dipole moments in the $(x, z)$-plane $(x \|[100])$ of $\beta-\mathrm{Ga}_{2} \mathrm{O}_{3}$ for various UV electronic (excitonic) transitions (top row) and different IR phonon modes (bottom row). Labels refer to notation in [80]. The left (right) column displays experimental (theoretical) results.

\section{Singular Axes}

3.1 Spectral Dispersion The analysis of the dielectric function of $\mathrm{Ga}_{2} \mathrm{O}_{3}$ put forward in [42] allows to calculate the angular position of the singular axes. In general, the complex dielectric tensor of orthorhombic, monoclinic and triclinic crystals leads to four singular axes. Such axes were first considered for orthorhombic material $[8,30]$ and have been named Windungsachsen initially [8] because their eigenstates are circularly polarized. The analytical treatment of the related problem from Maxwell's equations shows that orthorhombic, monoclinic and triclinic crystals can generally be distinguished optically from the degeneracy of the (complex) indices of refraction for these axes, exhibiting a single, 2 or 4 different values, respectively [42] (see Table 1). The three uniaxial crystal systems (tetragonal, hexagonal, trigonal) cannot be distinguished optically since they possess the same form of the dielectric tensor and remain uniaxial in the absorption regime [42]. We note that also assemblies of nanocrystals [92] or rare-earth doped crystals $[39,93]$ represent examples of (effective) birefringent absorptive media. Analytical solutions for orthorhombic and monoclinic crystals can be found in [42]; we point to previous discussions of the orientational dependence of the complex index of refraction $[94,95]$.

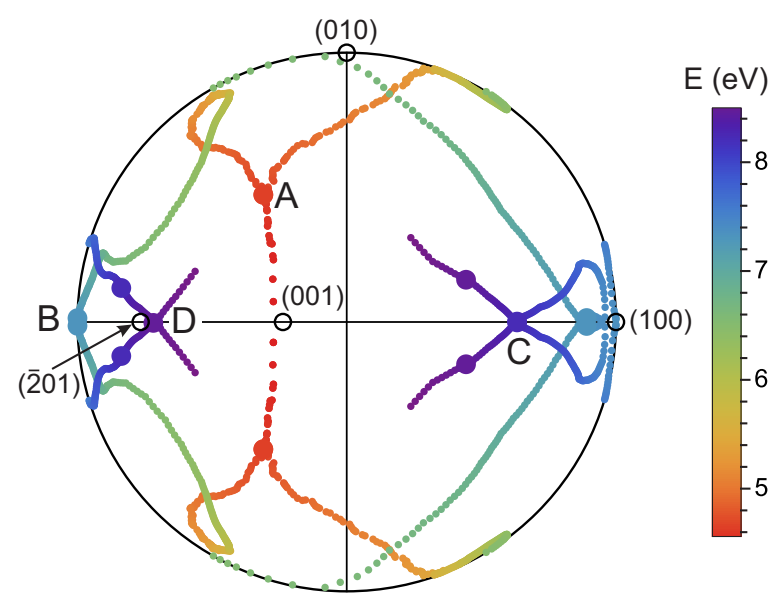

Figure 4 Stereographic projection of the angular positions of the singular axes in $\beta-\mathrm{Ga}_{2} \mathrm{O}_{3}$ as a function of photon energy. Crystallographic planes (marked by open circles) are labelled.

In Fig. 4 the spectral dispersion of the direction of the singular axes in monoclinic $\mathrm{Ga}_{2} \mathrm{O}_{3}$ is depicted. We note that the angular position of optic axes over a wider spectral range (in the transparency regime) has been reported previously, e.g. [96], but Ref. [42] represents the first report of the spectral dispersion of singular axes (in the absorption regime). The $(x, z)$ symmetry plane perpendicular to the [010]- $(y-)$ direction is obvious. At the onset of absorption (point 'A' in Fig. 4, $E=4.78 \mathrm{eV}$ ) the two optic axes from the transparency regime start to exhibit significant splitting into the four singular axes. 'B' denotes a range $(E \approx 7.23-7.33 \mathrm{eV})$ of "almost" uniaxial degeneracy close to the [100]-direction. At two spectral points (points 'C', $E=8.14 \mathrm{eV}$ and 'D', $E=8.37 \mathrm{eV}$ ) one pair of singular axes degenerates, forming a "normal" (but absorptive) optic axis, rendering the material triaxial [42], a case also considered in [41]. A more detailed discussion can be found in [42]. 


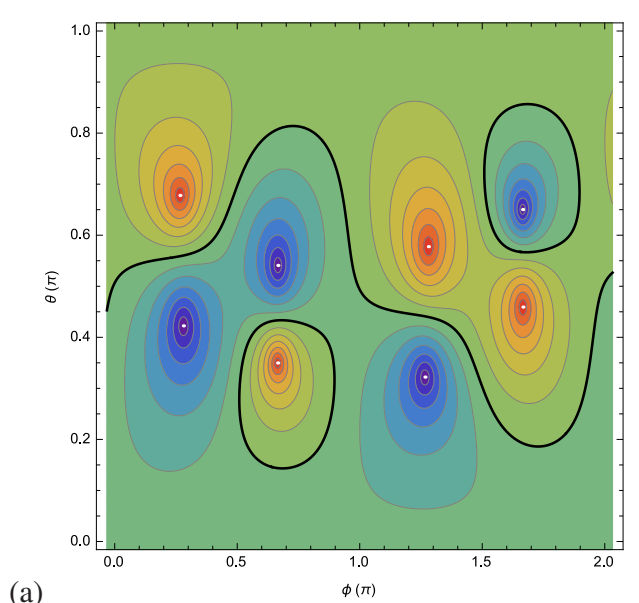

(a)

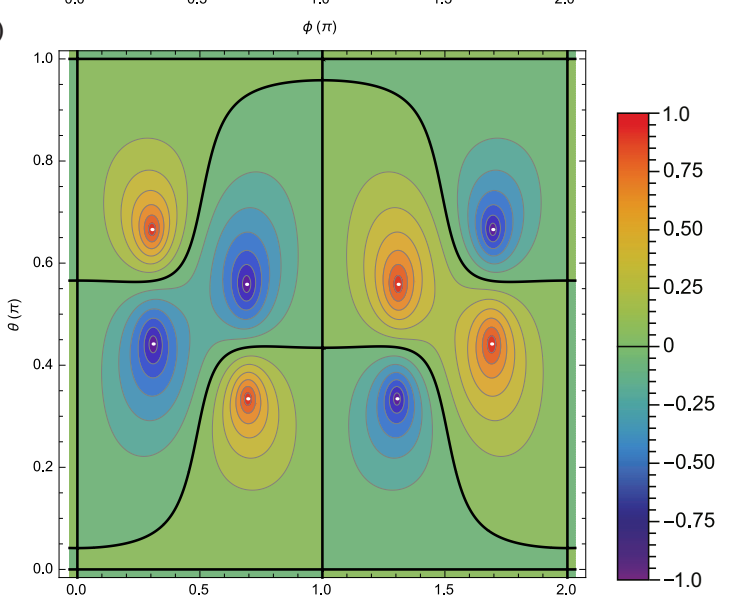

(b)

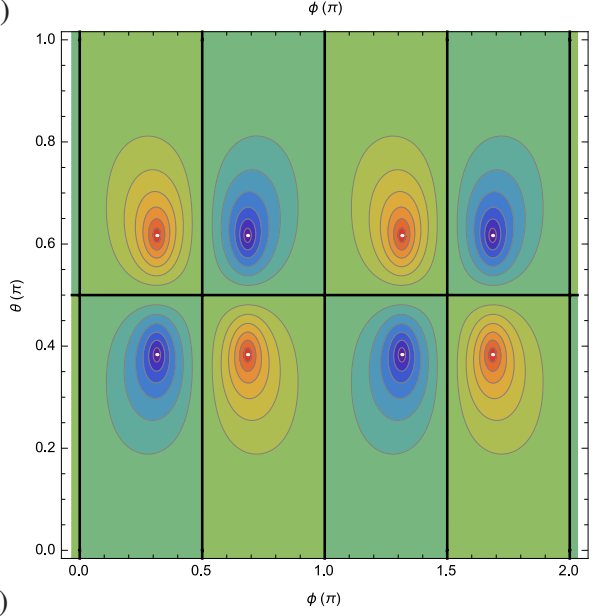

(c)

Figure 5 The Stokes vector component $S_{3}$ (circular polarization) in the $(\phi, \theta)$-plane (in units of $\pi$ ) in false colors. The singular axes are at $\left|S_{3}\right|=1$. The contour with $S_{3}=0$ (linear polarization) is shown as thick black lines. (b) depicts the situation for monoclinic $\left(\epsilon_{x z} \neq 0\right) \beta-\mathrm{Ga}_{2} \mathrm{O}_{3}$ at photon energy $E=6.20 \mathrm{eV}$. (a) depicts a fictitious triclinic material with the same dielectric tensor but additionally $\epsilon_{x y}=\epsilon_{x z}$. (c) depicts a fictitious orthorhombic material with the same dielectric tensor but $\epsilon_{x z}=0$. The white dots indicate the angular position of the singular axes.
3.2 Eigenstates The angular distribution of the circular polarization component of the eigenmodes (Stokes vector component $S_{3}$ ) is depicted in Fig. $5 \mathrm{~b}$ for $\beta-\mathrm{Ga}_{2} \mathrm{O}_{3}$ at a particular photon energy $(E=6.20 \mathrm{eV})$ [97]. The angles $\theta$ and $\phi$ denote the rotation of the crystal around the $y$ - and $z$-axis, respectively, as defined in [42]. At the position of the singular axes, it is \pm 1 , along the thick black lines it is zero (linear polarization). In terms of the notation of [98-100] the singular axes are 'C-points' and the lines of linear polarization are 'L-lines'.

The additional symmetry changes for a biaxial material that is less symmetric and triclinic (Fig. 5a) or more symmetric and orthorhombic (Fig. 5c) is shown for comparison, by setting $\epsilon_{x y}=\epsilon_{x z}$ and $\epsilon_{x z}=0$, respectively, for a fictitious similar material.

In Fig. 6 the situation is depicted for the crystallographic $y$-direction. At $E=6.59 \mathrm{eV}$, the Stokes vector component is $S_{3}=-0.997$, i.e. the direction represents 'almost' a singular axis. The spectral dependence of the projection of the Stokes vector on the $\left(S_{1}, S_{2}\right)$-plane is shown in Fig. 6a. In Fig. 6b, the spectral dependence of $S_{3}$ is shown together with the real and imaginary part of the index of refraction and $\left|n_{1}-n_{2}\right|\left(n_{1}\right.$ and $n_{2}$ refer to the complex indices of the two eigenmodes for a given direction and energy). At the energy $E=6.59 \mathrm{eV}$ the real parts of $n$ are identical while the imaginary parts of $n$ are only close. A more detailed discussion and Stokes vector analysis for eigenstates in other directions can be found in [42].

The change of the eigenvectors of the (transverse) fields $D_{a}$ and $D_{b}$ from orthogonal to parallel (at exactly the singular axis, $D_{a}$ and $D_{b}$ are degenerate (coalesced) and circularly polarized) is continuous. This can be seen from the (absolute value of the complex) scalar vector product (dot product) $\boldsymbol{D}_{b}{ }^{*} \cdot \boldsymbol{D}_{\boldsymbol{a}}$ as depicted in Fig. 7 . Its maximum at the singular axes is conical. For the case $\Im \epsilon=0, D_{b}{ }^{*} \cdot D_{a}$ vanishes for all orientations, which is well known. We note that non-orthogonal states that are not completely coalesced $\left(0 \leq \boldsymbol{D}_{\boldsymbol{b}}{ }^{*} \cdot \boldsymbol{D}_{\boldsymbol{a}} \leq 1\right)$ correspond to elliptically polarized states (cmp. Figs. $\overline{5 b}$ and $7 \mathrm{a}$ ).

For the absorption case, the polarizing properties of the biaxial crystal change with orientation from a homogeneous Jones matrix (with orthogonal eigenvalues) to an inhomogeneous Jones matrix, described with the inhomogeneity parameter $\eta=\left|\boldsymbol{E}_{\boldsymbol{b}}{ }^{*} \cdot \boldsymbol{E}_{\boldsymbol{a}}\right| \leq 1$ as defined in [101]. We note that the orientational dependence of $\eta$ looks practically identical to Fig. 7a.

3.3 Complex Index of Refraction The angular analysis of the two complex indices of refraction shows that besides $\Re n_{1}-\Re n_{2}=0$ and $\Im n_{1}-\Im n_{2}=0$ at the angular positions of the singular axes, $\Re n_{1}-\Re n_{2}$ is zero along continuous angular arcs limited by two singular axes (Fig. 8a) and $\Im n_{1}-\Im n_{2}$ vanishes along different arcs, connecting two different singular axes (Fig. 8b). The $\Re n_{1}-\Re n_{2}=0$ arcs run in the vicinity of the related optic axis for the case $\Im \epsilon=0$ (cmp. also Fig. 11e) 


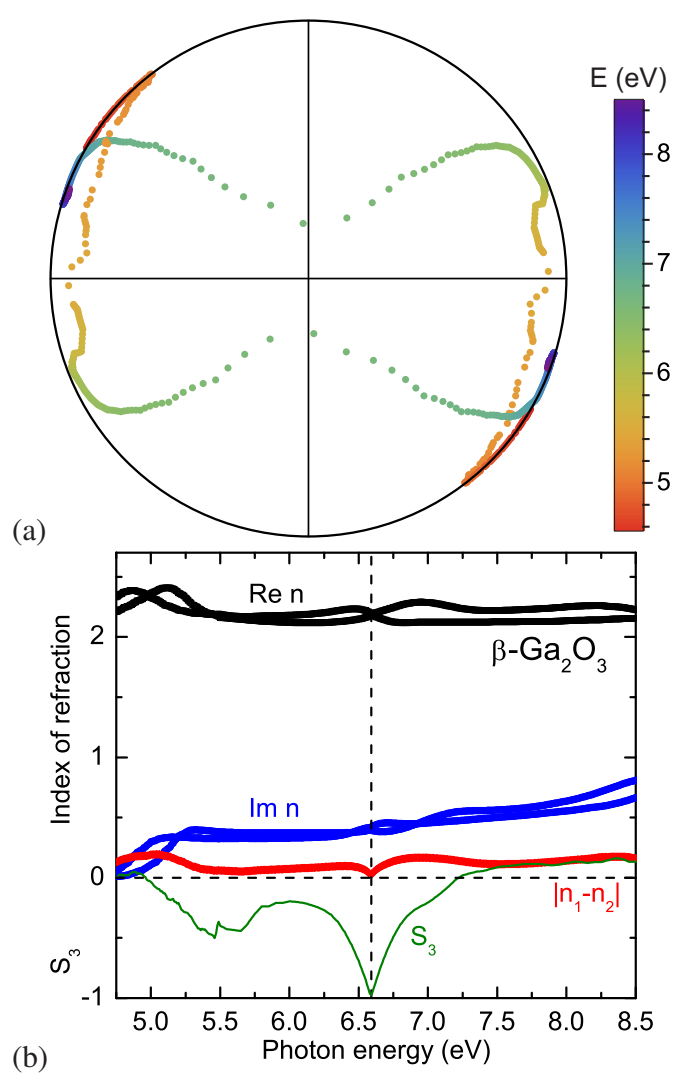

Figure 6 (a) Energy dependence of the Stokes vector (stereographic projection $\left(S_{1}, S_{2}\right), S_{3}= \pm 1$ is in the center of the graph) for the $y$-direction ([010]) (same color scale as in Fig. 4). (b) Spectral dependence of the real and imaginary parts of the index of refraction, their absolute difference and of the Stokes vector component $S_{3}$ for this direction. The energy $E=6.59 \mathrm{eV}$, where $\left|n_{1}-n_{2}\right|$ is close to zero and $S_{3}$ is close to -1 , is denoted with a vertical dashed line.

as pointed out in [28]. The $\Im n_{1}-\Im n_{2}=0$ arcs run in the vicinity of the related "absorption" axis for the case $\Re \epsilon=0$. Another visualization of this situation are the three-dimensional representations of $\Re n$ and $\Im n$ in Fig. 9a,b which look as described by Drude [102] like "eine Fläche mit zwei Schalen [..., die] längs gewisser Kurvenstücke, die bei monoklinen und triklinen Krystallen unsymmetrisch zu den Coordinatenebenen liegen, zusammenhängen.” [103]. A modern theory of this phenomenon is formulated with Hamiltonians in [104]. It was shown for (monoclinic) $\mathrm{Nd}: \mathrm{YCOB}\left(\mathrm{YCa}_{4} \mathrm{O}\left(\mathrm{BO}_{3}\right)_{3}\right)$, prepared in the form of a spherical crystal, a geometry already studied for ruby [105] and used by Raman [106], that there exists an angular range for which the absorption coefficient is independent of the polarization [39].

For comparison the situation for artificially setting $\Im \epsilon=0$ is depicted in Fig. 9c,d, displaying the double cone (diabolic) structure around one of the (two conventional) optic axes. We note that a similar structure has

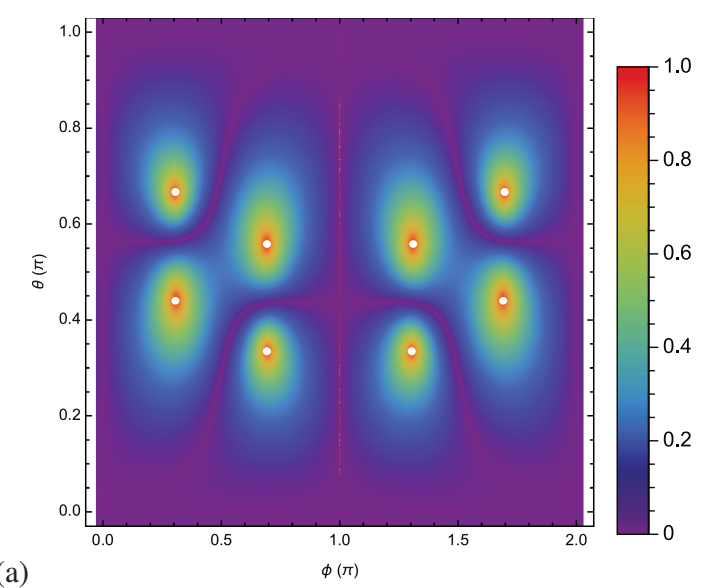

(a)

(b)

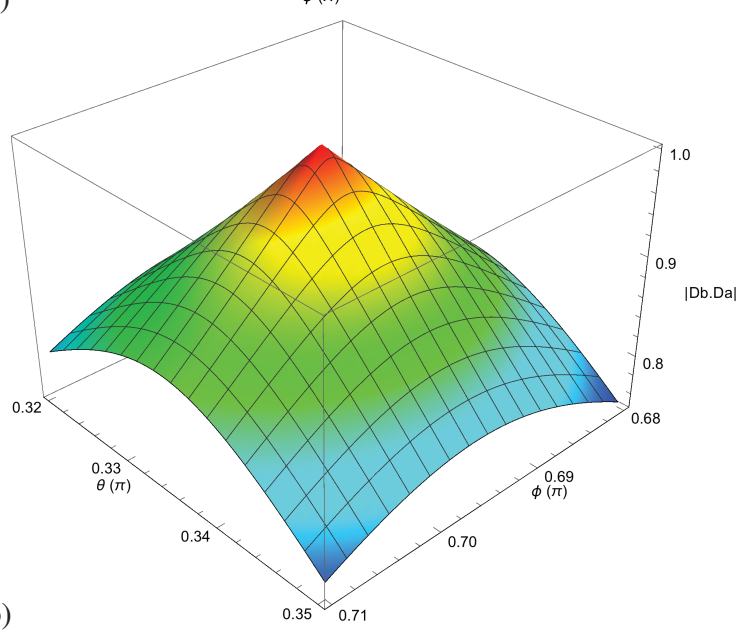

Figure 7 (a) The dot product $\left|\boldsymbol{D}_{b}{ }^{*} \cdot \boldsymbol{D}_{\boldsymbol{a}}\right|$ of the eigenvectors; while small for most directions, it becomes 1 on the singular axes. The white dots indicate the angular positions of the singular axes. (b) $3 \mathrm{D}$ visualization around one of the singular axes.

been found for heavy and light hole dispersions in strained materials [107].

In Fig. 10 the typical bifurcations [108] at an exceptional point are shown by varying artificially the imaginary part of the dielectric function. Also the analogue exceptional point for negative imaginary part appears $(\gamma=-1$ in Fig. 10a) that would occur in the presence of gain.

3.4 Reflection and Transmission The problem of reflection and transmission of circularly polarized waves from a crystal cut perpendicular to a singular axis has already been brought up in [29]. In the Appendix 7.1 the current treatment of reflection (and transmission) from a planar interface of a biaxial material is sketched and the result shall be shown here for $\beta-\mathrm{Ga}_{2} \mathrm{O}_{3}$ at the energy $E=$ $6.20 \mathrm{eV}$. The reflection coefficients for circularly polarized light, defined via

$$
\left(\begin{array}{c}
l_{r} \\
r_{r}
\end{array}\right)=\left(\begin{array}{ll}
R_{l l} & R_{l r} \\
R_{r l} & R_{r r}
\end{array}\right)\left(\begin{array}{c}
l_{i} \\
r_{i}
\end{array}\right),
$$


(a)
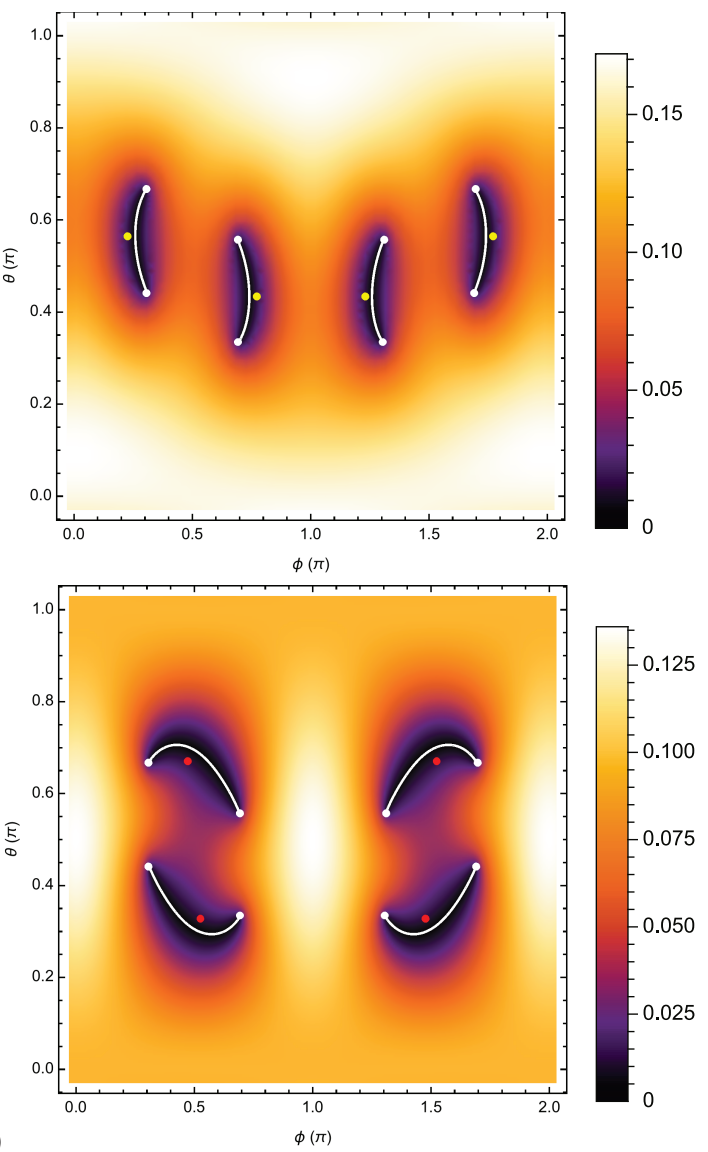

(b)

Figure 8 The difference of the two indices of refraction as (a) $\left|\Re n_{1}-\Re n_{2}\right|$ and (b) $\left|\Im n_{1}-\Im n_{2}\right|$ as a function of propagation direction for $\beta-\mathrm{Ga}_{2} \mathrm{O}_{3}$ at a photon energy of $E=6.20 \mathrm{eV}$. The white lines indicate the zeros, the white dots denote the angular positions of the singular axes, the yellow (red) dots the related optic axes for the case $\Im \epsilon=0(\Re \epsilon=0)$.

where $l_{i}, r_{i}\left(l_{r}, r_{r}\right)$ denote the incoming (reflected/outgoing) left- and right-circular polarized waves, are depicted (absolute values) in Fig. 11a-c.

A left-circularly polarized wave is mostly reflected as right-polarized wave and vice versa, the related reflection coefficients are termed $R_{l r}$ and $R_{r l}$ and are found identical for all angles (Fig. 11a). A small part suffers mode conversion and comes back for incoming left-circular wave as left-circular reflected wave, its reflectivity denoted by $R_{l l}$ (Fig. 11b); $R_{r r}$ is defined accordingly (Fig. 11c). For each of the left or right incoming waves four singular directions lead to fully circularly polarized light (either $R_{l l}$ or $R_{r r}$ are zero and $\left|S_{3}\right|=1$ ). In particular, when a left-circular wave $\left(S_{3}=-1\right)$ falls onto a singular axis with $S_{3}=+1$ (cmp. Fig. 5b), $R_{l l}=0$ and the reflected wave is purely right-circular $\left(S_{3}=+1\right)$. If (artificially) the absorption is set to zero ( $\Im \epsilon=0), R_{l l}=R_{r r}$ with its zeros on the optic axes as visualized in Fig. 11d-f.
We note that the exact angular positions of the singular axes are strictly never numerically evaluated. However, the eigenvectors of the (transverse) fields $D_{a}$ and $D_{b}$, i.e. $\boldsymbol{\epsilon} \boldsymbol{E}_{\boldsymbol{a}}$ and $\boldsymbol{\epsilon} \boldsymbol{E}_{\boldsymbol{b}}$ with the fields in (9), change continuously from orthogonal to parallel (at exactly the singular axis $\boldsymbol{D}_{\boldsymbol{a}}$ and $D_{b}$ are degenerate) (cmp. Fig. 7). We note that at $e x$ actly the singular axis the equations (14) and (15) are undetermined, but already in the vicinity of the singular axes, the eigenvectors are non-orthogonal and the transmission coefficients derived from the boundary conditions (14) and (15) are unphysical.

Instead a matrix approach for the propagation (Appendix 7.2) can be used that completely avoids the (technically unnecessary) vector decomposition into eigenstates within the biaxial medium. The electric field entering the biaxial medium is subject to "unconventional" propagation (see next section). The results for the reflection coefficients, as depicted in Fig. 11, remain valid and for the transmission physically reasonable values are obtained.

3.5 Propagation From the previous section, it is clear that a left- and a right-circularly polarized wave can enter the biaxial crystal when they fall onto a singular axis. Let us look at a singular axis that is right-polarized $\left(S_{3}=+1\right)$. Then the polarization of the right-circular wave, which is the eigenstate, remains unchanged while the wave propagates through the crystal (Voigt wave, Fig. 12a). The left-circular wave is asymptotically converted into a rightcircular wave as also depicted in Fig. 12a. From Fig. 12b it can be seen that left-circular wave actually suffers the smaller absorption. This behavior has been already discussed in [32,109] and experimental results have been reported for $\mathrm{KGd}\left(\mathrm{WO}_{4}\right)_{2}: \mathrm{Nd}$ [110]. Here it becomes clear that the left-circular fraction suffers the same attenuation as the right-circular, i.e. $T_{l l}=T_{r r}$, their definition being similar to (4),

$$
\left(\begin{array}{c}
l_{t} \\
r_{t}
\end{array}\right)=\left(\begin{array}{cc}
T_{l l} & T_{l r} \\
T_{r l} & T_{r r}
\end{array}\right)\left(\begin{array}{c}
l_{i} \\
r_{i}
\end{array}\right)
$$

$l_{t}, r_{t}$ denoting the circularly polarized transmitted waves; for the calculation of the matrix elements in (5) the matrix $T$ (28) from Appendix 7.2 is used. For the left-circular incoming wave additionally a right-circular wave exists $\left(T_{r l} \neq 0\right)$ which enhances the transmittance for the initially left-circular wave (Fig. 12b). We note that since the rightcircular wave passes without mode conversion, $T_{l r}=0$. At the thickness $(d \approx 0.537 \mu \mathrm{m})$ where $S_{3}=0$, also $T_{l l}=T_{r l}$.

In Fig. 13 shows the Stokes vector of transmitted light propagating in the crystallographic $y$-direction at $E=$ $6.59 \mathrm{eV}$ when this direction is close to a $S_{3}=-1$ singular axis (cmp. Fig. 6). Mode conversion occurs for both left- and right-circular waves but the incident left-circular polarized light remains at least within the range of negative $S_{3}$. 
(a)

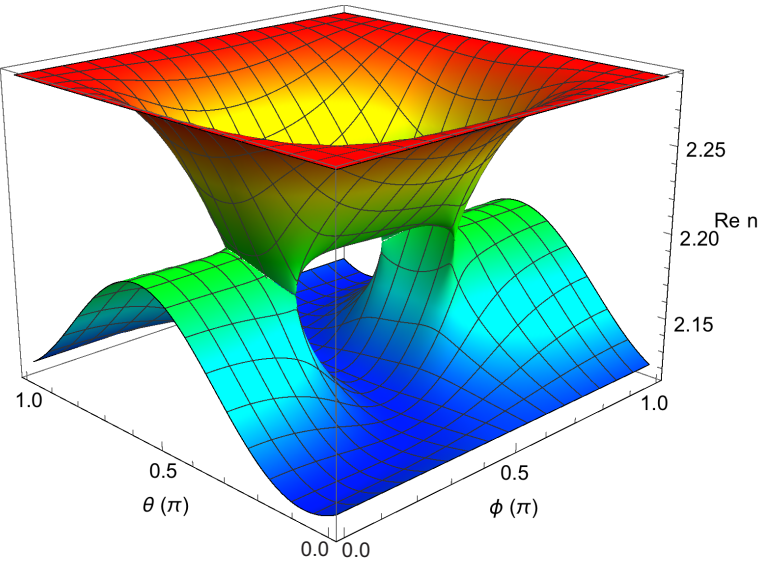

(c)

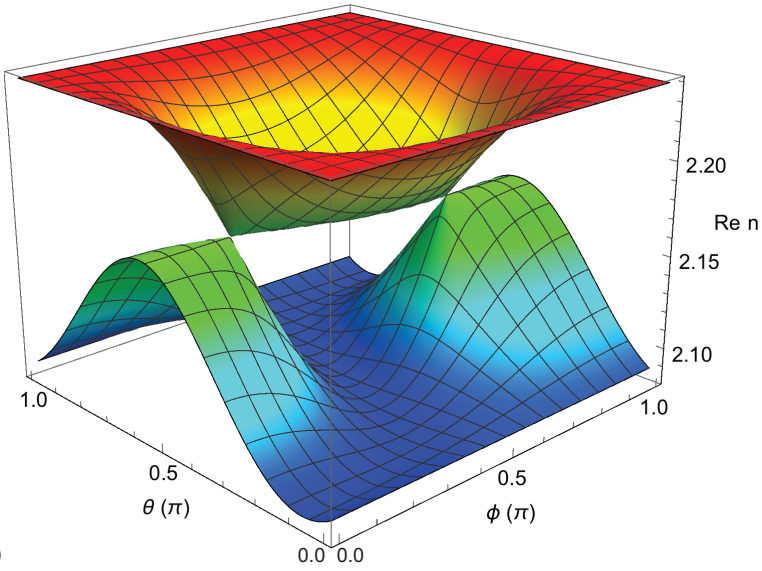

(d)

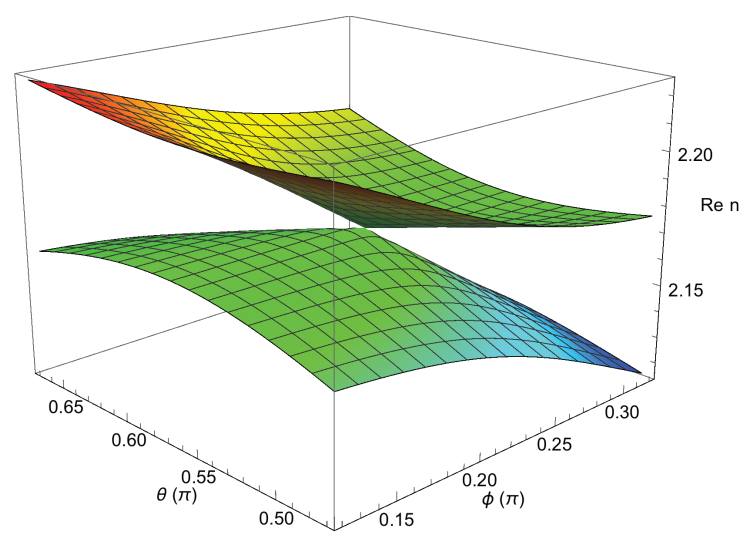

Figure 9 Three-dimensional plot of (a) $\Re n$ and (b) $\Im n$ as a function of propagation direction (for clarity only for the hemisphere $0 \leq \phi \leq \pi)$ for $\beta-\mathrm{Ga}_{2} \mathrm{O}_{3}$ at a photon energy of $E=6.20 \mathrm{eV}$. The connections of the upper and lower branch correspond to the white lines in Fig. 8. (c) Three-dimensional plot of $n=\Re n$ when the imaginary part of the dielectric function for $\beta$ - $\mathrm{Ga}_{2} \mathrm{O}_{3}$ at a photon energy of $E=6.20 \mathrm{eV}$ is artificially set to zero as a function of propagation direction. (d) Same as (c) but zoomed to one of the optic axes, displaying the diabolic point.

(a)

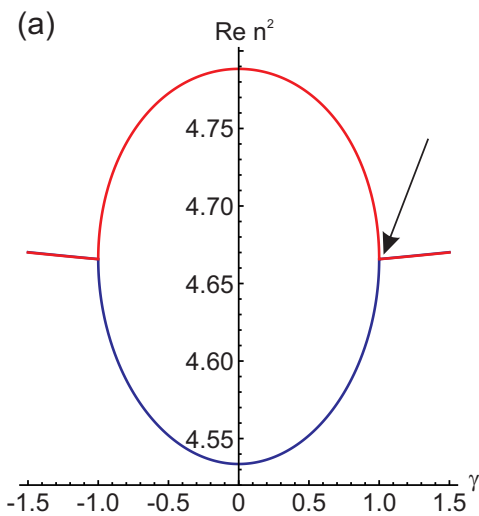

(b)

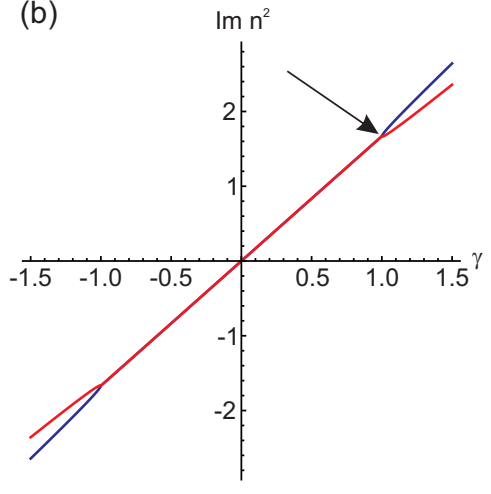

(c) $\operatorname{Im~} n^{2}$

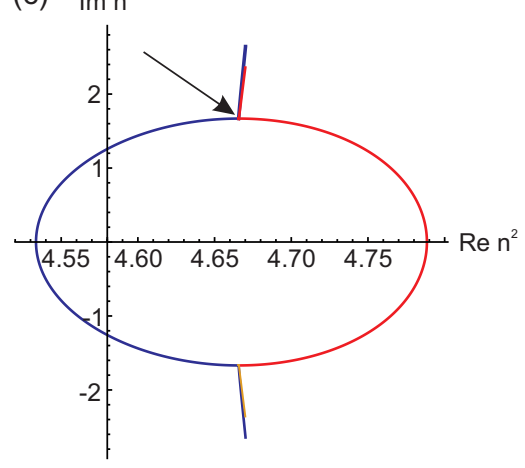

Figure 10 (a) Real and (b) imaginary values of the squares of the indices of refraction for the orientation of a singular axis (same as in Fig. 7) in $\beta-\mathrm{Ga}_{2} \mathrm{O}_{3}$ at a photon energy of $E=6.20 \mathrm{eV}$ and setting artificially the dielectric function to $\tilde{\epsilon}=\Re \epsilon+\gamma \Im \epsilon$. (c) depicts the two indices in the complex plane depending parametrically on $\gamma \cdot \gamma=1$ represents the actual dielectric function and the exceptional point is indicated by the arrows; negative values of $\gamma$ or $\Im n^{2}$ denote gain regime.

\section{Planar microcavities with anisotropic media} A planar microcavity is a layered system consisting of a cavity medium (typically of thickness $(2 n+1) \times \lambda / 2$, 

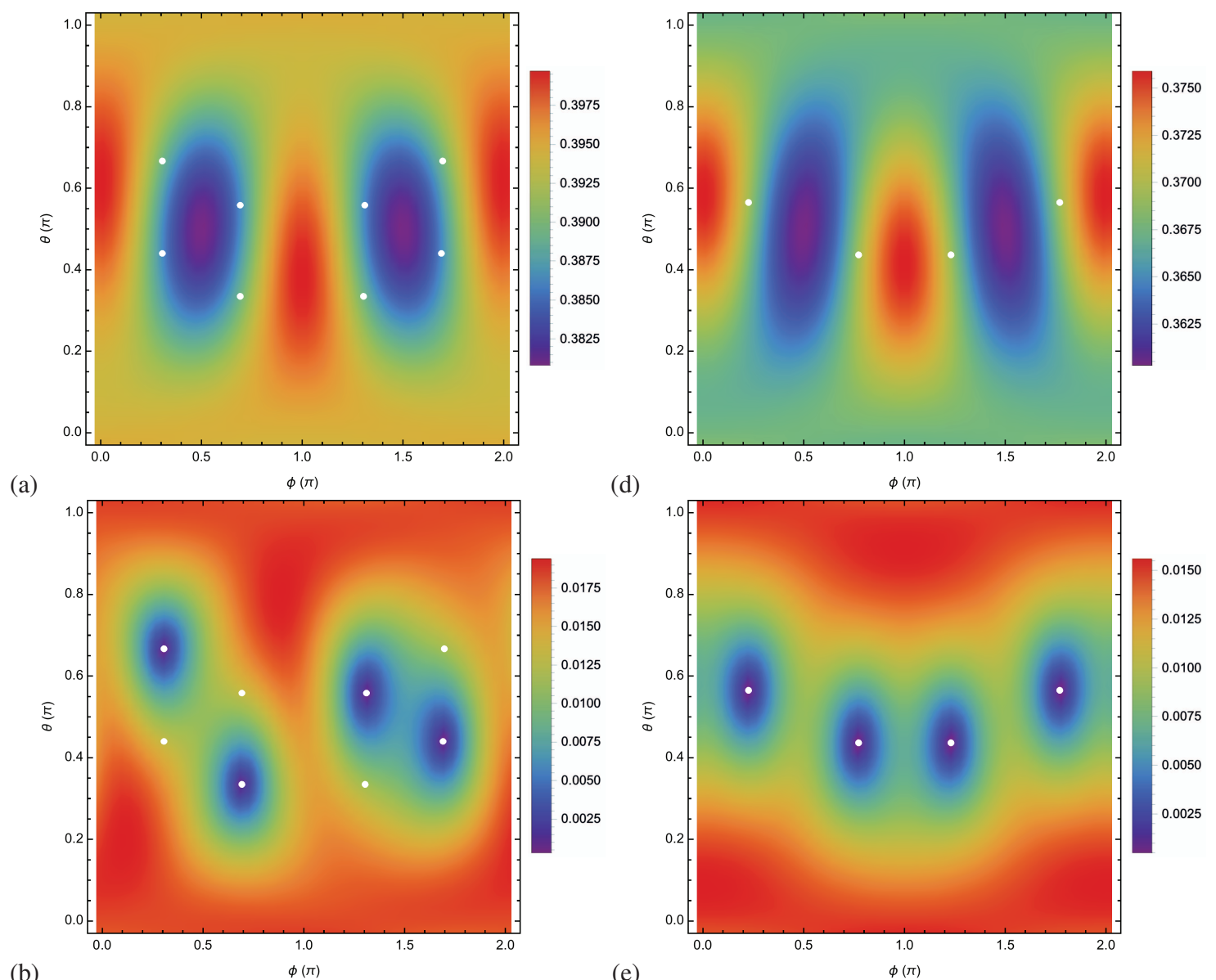

(d)
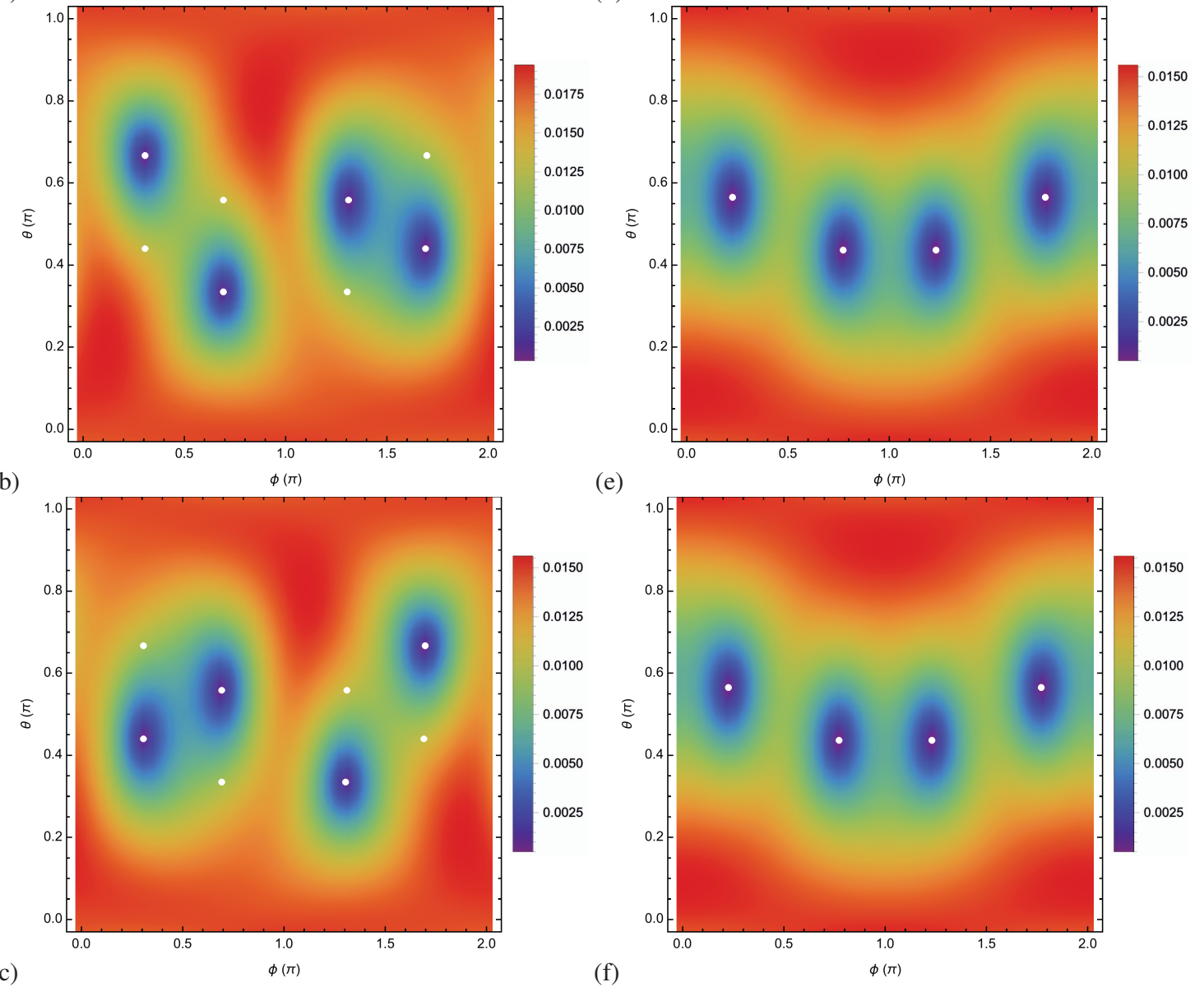

Figure 11 The reflection coefficients of circularly polarized light for normal incidence from $\beta-\mathrm{Ga}_{2} \mathrm{O}_{3}$ at $E=6.20 \mathrm{eV}$, (absolute values) (a) $R_{l r}=R_{r l}$, (b) $R_{l l}$ and (c) $R_{r r}$. The white dots indicate the angular positions of the singular axes. (d)-(f) show the same quantities for setting (artificially) $\Im \epsilon=0$ (leading to $R_{l l}=R_{r r}$ ); in this case the white dots indicate the angular positions of the conventional optic axes.

$n=0,1, \ldots)$ sandwiched between two distributed Bragg reflector (DBR) mirrors. This way the growth direction per se introduces a preferred direction and rotational symmetry. The dispersion of the cavity mode is a fundamental property of such cavity. In [111] the effect of an uniaxial cavity material and the orientation of its optic axis relative to the cavity axis was investigated theoretically. If the optic axis and the cavity axis are aligned no particular effects are 

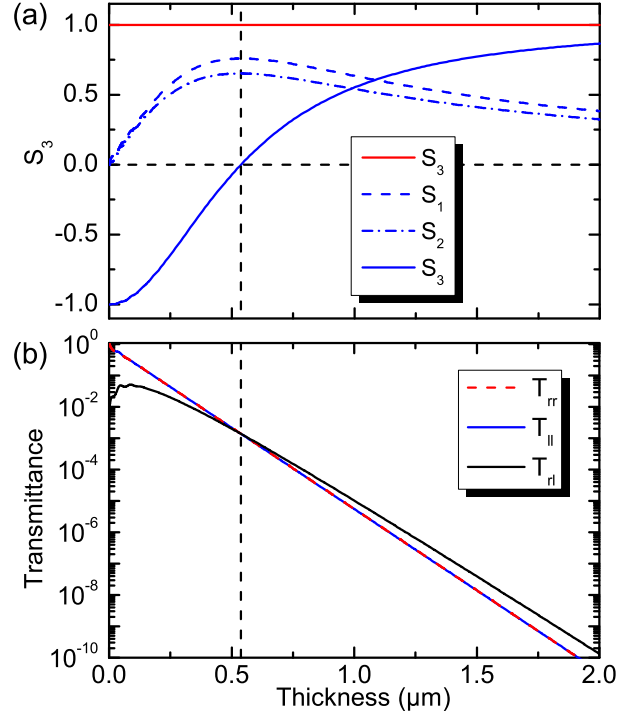

Figure 12 (a) The Stokes vector of the transmitted light (normal incidence, $E=6.20 \mathrm{eV}$ ) from a $\mathrm{Ga}_{2} \mathrm{O}_{3}$ slab cut perpendicular to a $S_{3}=+1$ singular axis (same as in Fig. 7) as a function of the slab thickness for incoming right- (left-) circularly polarized light shown as red solid (blue solid) curve. For incoming leftcircular light, the Stokes vector components $S_{1}$ and $S_{2}$ are shown as dashed and dashed-dotted lines, respectively. (b) Transmitted intensity for right-circular light $\left(T_{r r}\right.$, red curve $)$ and left-circular light exiting as left-circular $\left(T_{l l}=T_{r r}\right.$, blue curve $)$ and right circular $\left(T_{r l}\right.$, black curve). We note that $T_{l r}=0$. The vertical dashed line indicates the thickness for which $T_{l l}=T_{r l}$.

present. For a general orientation of the optic axis, when it is tilted with respect to the stacking direction, the rotational symmetry of the system does not hold anymore. In this case the medium contains two distinguished directions and is effectively biaxial.

This results at least in the TE- and TM-polarized cavity-photon mode(s) not coinciding at normal propagation angle (Fig. 14). Such effect has been observed experimentally for microresonators with nonpolar GaNbased [112-114] and ZnO-based [115] cavities (Fig. 15). The typical setup of such cavity is a homoepitaxial bottom

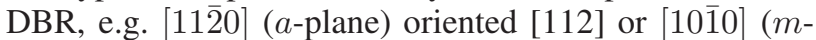
plane) oriented [113] (Al,Ga)N/GaN or $m$-plane oriented $(\mathrm{Mg}, \mathrm{Zn}) \mathrm{O} / \mathrm{ZnO}$ [115], a cavity with the wurtzite $c$-axis in-plane and a top mirror (dielectric or epitaxial); such system is effectively orthorhombic. Generally, also a tilted $c$-axis (unless parallel to the growth direction) results in a case of reduced symmetry. For the epitaxial growth of such structures, strain relaxation is important due to the lattice mismatch between the various layers due to the dependence of the lattice constants of $\left(\mathrm{Al}_{x} \mathrm{Ga}_{1-x}\right) \mathrm{N}$ or $\left(\mathrm{Mg}_{x} \mathrm{Zn}_{1-x}\right) \mathrm{O}$ on the alloy concentration. We note that the said nitride and oxide alloy systems behave rather differently since $\partial c / \partial x \times \partial a / \partial x$ is positive or negative, respectively [75].

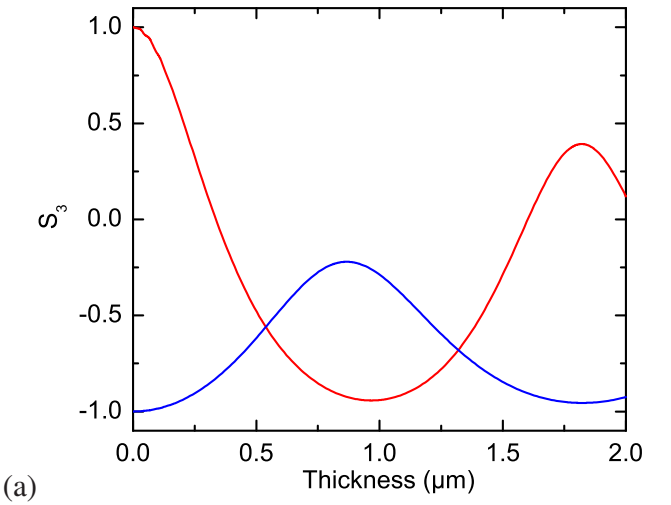

(a)

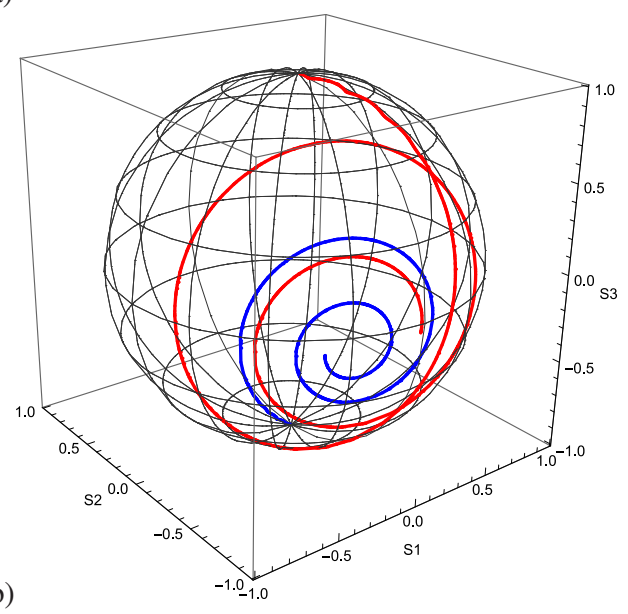

Figure 13 (a) Stokes vector component $S_{3}$ of the transmitted light (normal incidence, $E=6.59 \mathrm{eV}$ ) from a $\mathrm{Ga}_{2} \mathrm{O}_{3}$ slab cut perpendicular to the $y$-axis (cmp. Fig. 6) as a function of the slab thickness $d$ for incoming right- (left-) circularly polarized light shown as red (blue) curves. (b) Stokes vector of transmitted light for incoming right- (left-) circularly polarized light shown as red solid (blue solid) curves and $d=0-4 \mu \mathrm{m}$.

Based on a Maxwell approach to the propagation of electromagnetic waves with complete polarization treatment, complex mode energies can be numerically determined as singularities of the system and the respective polarization is obtained from the matrix kernel [116]. A cavity photon mode is found to be split into two modes, whose polarization states are generally elliptical and thus nonorthogonal to each other. Depending on the cavity thickness, those two modes can be degenerate (same energy and broadening) at finite in-plane wave vectors which correspond to propagation nearly but not exactly along or perpendicular to the projection of the optic axis in the cavity plane. While the modes have linear orthogonal polarizations at zero in-plane wave vector (vertical emission), these degeneracy points are circularly co-polarized and represent exceptional points [117]. In particular, the degeneracy points of energy and broadening occur pair-wise (Fig. 16). The connecting line between them reveals a discontinuity of the mode broadening and polarization. Starting from the 


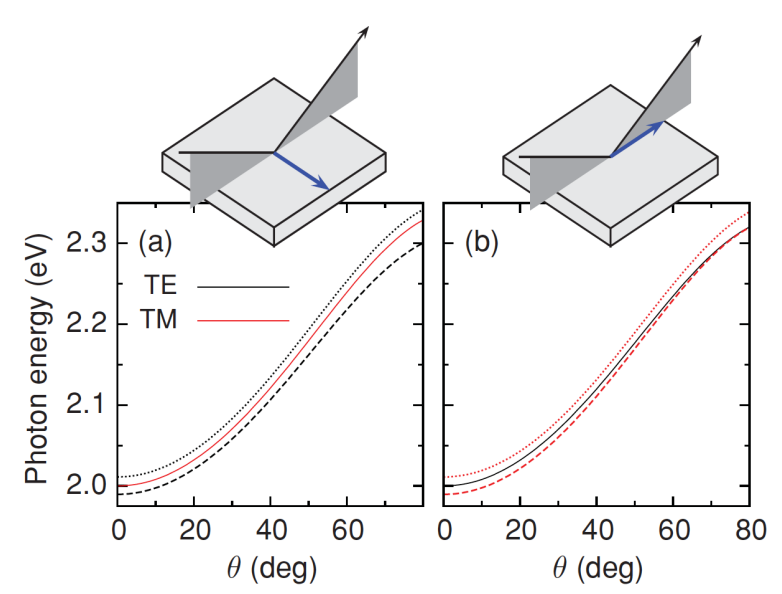

Figure 14 Dispersion versus propagation angle of the TE- (black lines) and TM- (red lines) polarized cavity-photon mode for two different directions of the optic axis of the uniaxial cavity material within the cavity plane. The cavity-photon mode which is connected to the ordinary wave within the cavity is shown as solid line whereas the extraordinary one is shown as dashed line (for positive birefringence) or as dotted line (for negative birefringence).

exceptional points, arcs of constant energy and broadening (shown as solid red lines in Fig. 16, cmp. Fig. 8) extend in the $\boldsymbol{k}_{\|}$-plane. The linear polarization is predicted to exhibit a vortex-like structure (Fig. 17) in the momentum space around the degeneracies [117].

For a m-plane $(\mathrm{Mg}, \mathrm{Zn}) \mathrm{O} / \mathrm{ZnO} \lambda$-microcavity we have measured polarized transmission (Fig. 15c). The Stokes vector as a function of energy and angle of incidence is depicted for two in-plane orientations of the $c$-axis (rotated by $\pm 30^{\circ}$ relatively to the situation in Fig. 14a). The finite circular component $\left(S_{3}\right)$ of about \pm 0.15 at its extrema indicates non-orthogonal, partially coalesced eigenstates as predicted. Further design of cavity parameters, in particular of the cavity thickness, should move the exceptional points into the light cone and make fully circularly polarized states observable.

We like to mention here that chiral modes at exceptional points have also been found for whispering gallery resonators $[118,119]$.

\section{Raman Scattering}

5.1 Treatment of Birefringence The problem of Raman scattering in anisotropic crystals has been realized early on [69] and the failure of the conventional approach in birefringent materials (off a principal axis) has been corroborated in [120] 50 years ago. The analysis of such Raman scattering intensities was deemed "pointless" [121] and the effect of birefringence "predictably catastrophic" [122]. However, we attribute this only to the lack of including correct crystal optics into Raman theory. The closest to a solution is the work of Rulmont et

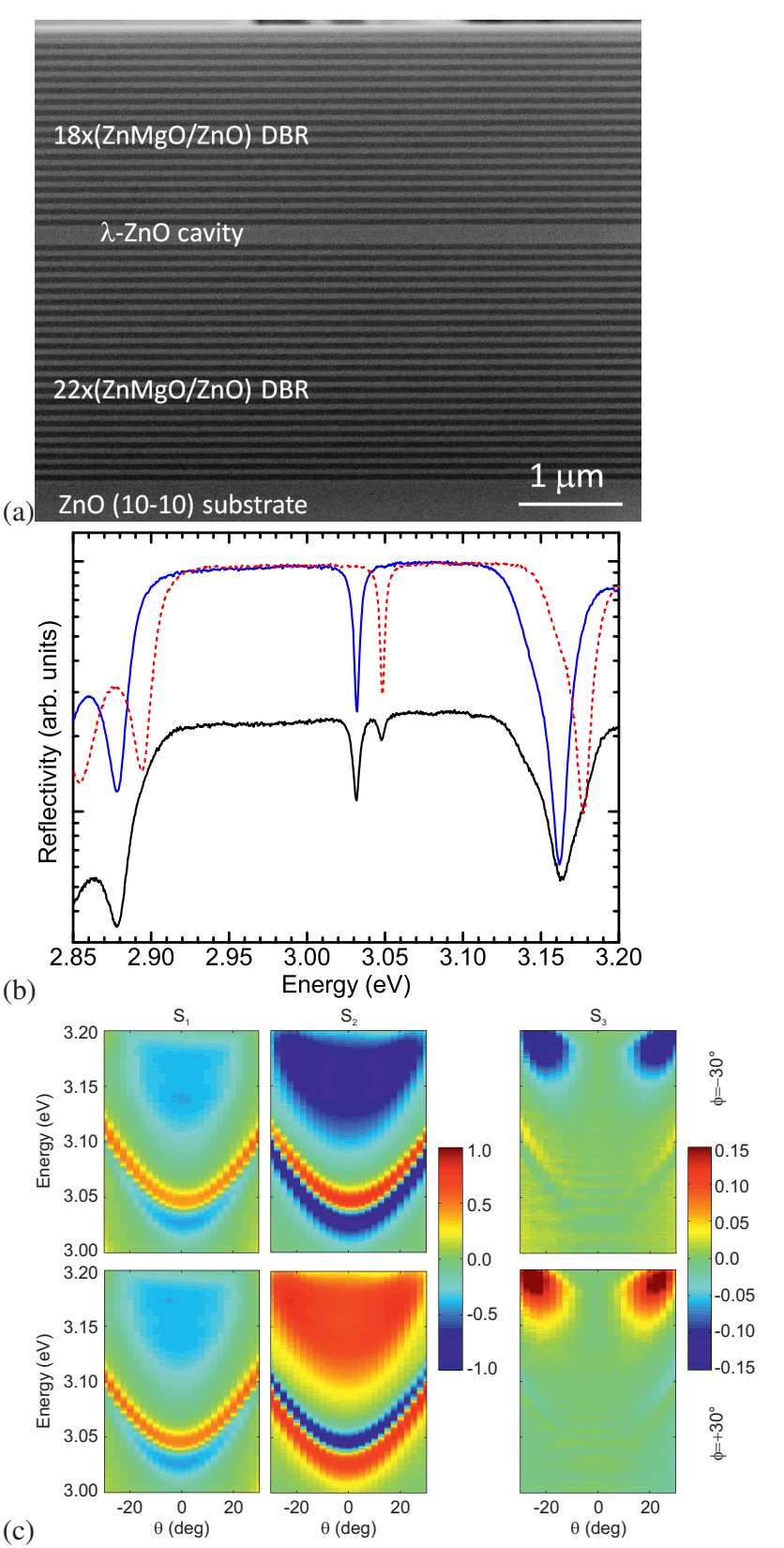

Figure 15 (a) Cross-section scanning electron microscopy image of $m$-plane $n_{1} \times\left[\mathrm{ZnO} / \mathrm{Mg}_{x} \mathrm{Zn}_{1-x} \mathrm{O}\right] \mathrm{DBR} / \mathrm{ZnO} / n_{2} \times$ $\left[\mathrm{ZnO} / \mathrm{Mg}_{x} \mathrm{Zn}_{1-x} \mathrm{O}\right]$ DBR microcavity $\left(n_{1}=18, n_{2}=22\right.$, $x \approx 0.22$ ). (b) Macroreflectivity measurements of a m-plane cavity ( $n_{1}=15, n_{2}=16$ ) for normal incidence, unpolarized (black, vertically shifted for clarity) and linear polarization resolved (red, polarization perpendicular to the $c$-axis, and blue parallel to the $c$-axis) spectra. (c) (Normalized) Stokes vector components for the angular dispersion of polarized transmission through a nonpolar m-plane $(\mathrm{Mg}, \mathrm{Zn}) \mathrm{O} / \mathrm{ZnO} \lambda$-microcavity. The $c$-axis is inplane and azimuthally rotated by $\phi= \pm 30^{\circ}$ as labelled with respect to the direction normal to the angular scan $\left(\phi=0^{\circ}\right.$ relates to the configuration in Fig. 14a). 

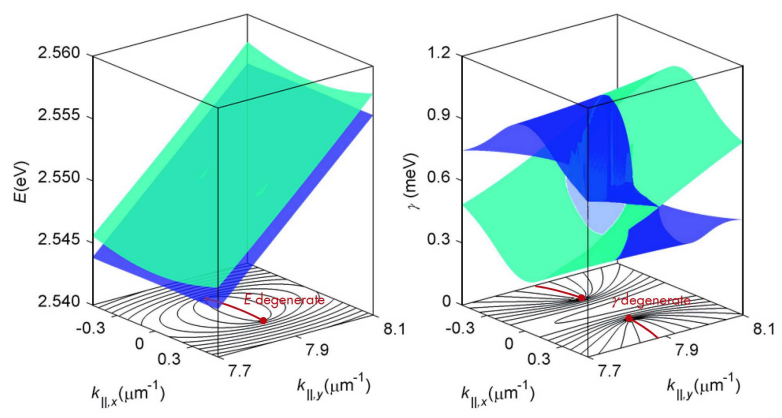

Figure 16 Dispersion of energy $E$ and broadening $\gamma$ depending on the in-plane wave vector $\boldsymbol{k}_{\|}$. The cavity is uniaxial with the optic axis along $y$ and the optical cavity thickness (along $z$ ) is larger than half the central wavelength of the surrounding Bragg mirrors. The mode degeneracies of $E$ and $\gamma$ are shown as red dots in the projection, the arcs of constant energy and broadening are shown as solid red lines.
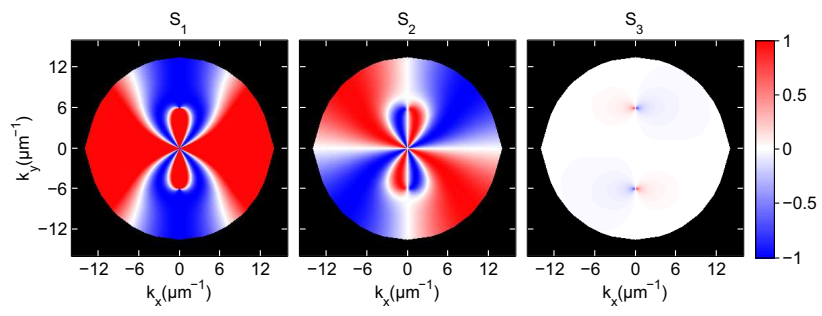

Figure 17 Polarization Stokes vector components of one cavity photon mode depending on the in-plane wave vector $\boldsymbol{k}_{\|}$. The cavity is uniaxial with the optic axis along $y$ and the optical cavity thickness (along $z$ ) is larger than half the central wavelength of the surrounding Bragg mirrors. The mode degeneracies are the four circularly polarized spots $\left(S_{3}\right)$ which are accompanied by a discontinuity of the linear polarization (see $S_{1}$ ).

al. [123,124]. In [125] the effect of birefringence on the Raman scattering from black phosphorus was modeled for a special scattering geometry. The concept of our reported theory [74] is to consider for a crystal of arbitrary symmetry the mode conversion from linear incoming polarization to varying elliptical/circular polarization along the path of the exciting radiation into the sample and likewise for the path of the scattered radiation back out of the sample. The Raman scattering event in between is correctly described (in the transparency regime) with a real-valued Raman tensor. Averaging is necessary over the excitation laser intensity distribution and excitation depth (or sample thickness). This scheme allows to determine the elements of the Raman tensor and their (relative) signs.

5.2 Uniaxial Crystals The effect of birefringence $\Delta n$ is visualized for (optically uniaxial) wurtzite $\mathrm{GaN}$ and $\mathrm{ZnO}$ in Fig. 18. Both Raman experiments have been executed similarly in the transparency regime $(532 \mathrm{~nm})$ on $a$-oriented single crystals. The response stems from within the focus depth which is large compared to $\lambda / \Delta n$; in this

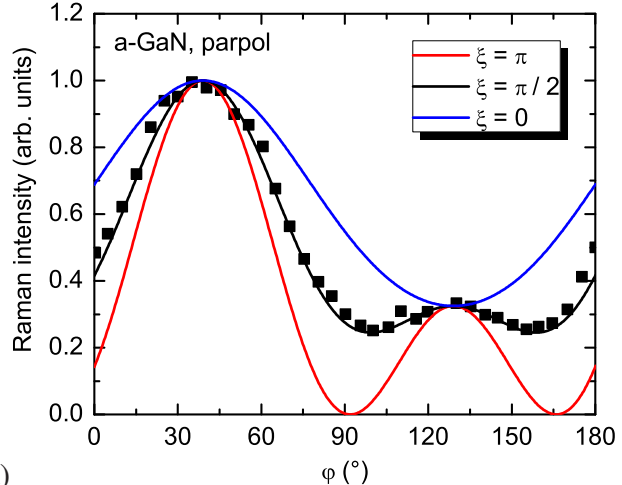

(a)

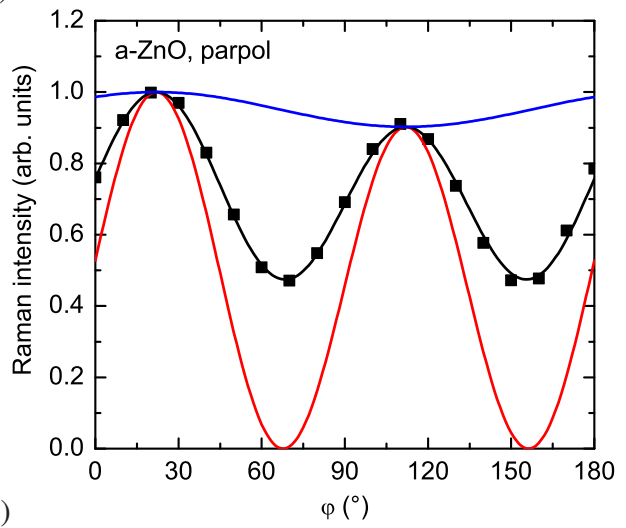

Figure 18 (a) Raman scattering intensity for a-oriented GaN $\left(\mathrm{A}_{1}\right.$-mode) in parallel-polarized configuration as a function of angle of incoming polarization. Experimental data (symbols) taken from [126] and fits (solid lines) for $\xi=0, \pi / 2$ and $\pi, \pi / 2$ describing obviously correctly the limit of large excitation depth. (b) Same for a- $\mathrm{ZnO}$, experimental data from [74].

case the apparent angle between (in short) the effective Raman tensor elements $a$ and $b$, which is more precisely defined in [74], is $\xi=\pi / 2$. This apparent angle has the same effect on the Raman intensity as the ad hoc phase parameters introduced in some publications [70-72], but has a strict physical meaning. An important and yet unexplored point is that for thin films, $\xi$ takes values between 0 (or $\pi$, depending of the relative signs of the tensor elements) and around $\pi / 2$ [74], and experiments allow to determine either the film thickness for known birefringence or $\Delta n$ for known film thickness.

5.3 Biaxial Crystals The approach with ad hoc phase parameters completely fails for optically biaxial crystals. We demonstrate this for monoclinic $\mathrm{Ga}_{2} \mathrm{O}_{3}$ [127]. Despite up to three additional free parameters introduced by the relative phases, the agreement to experimental data is worse than for using the physically correct model [128]. If different crystal cuts are considered, the discrepancy becomes even more striking since the ad hoc parameters cannot explain the different intensities observed for identical polarization directions (indicated by colored lines in Fig. 19). For known dielectric tensor at the excitation wavelength 
(010)
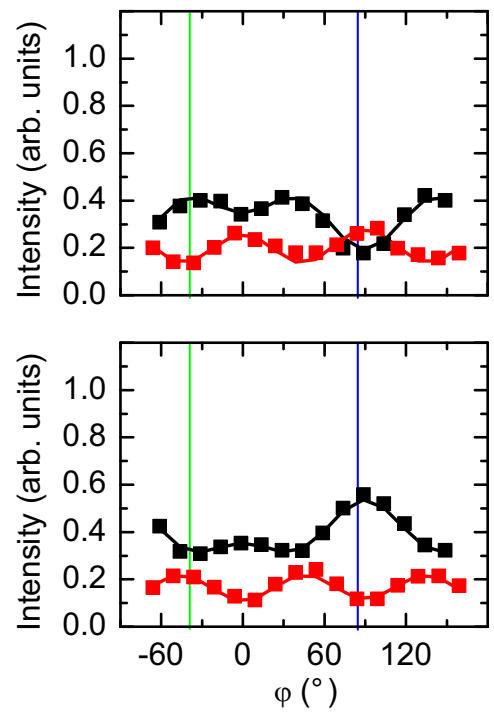

$(\overline{2} 01)$
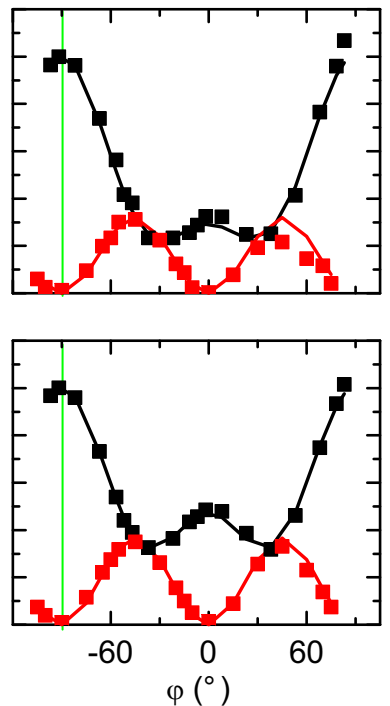

$(100)$
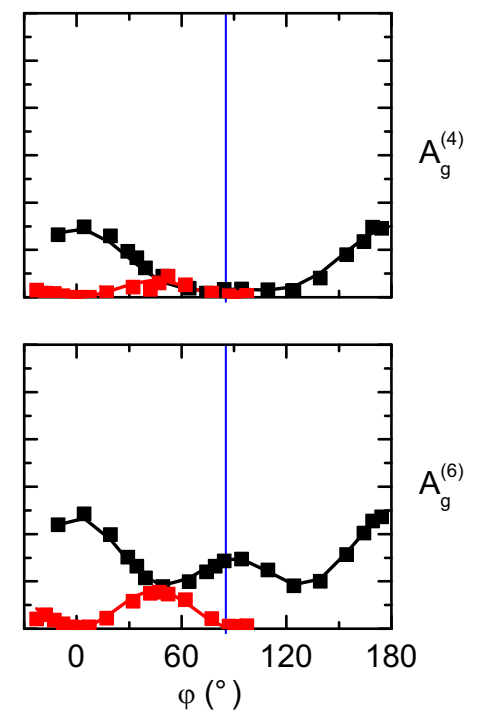

Figure 19 Raman scattering intensity for two selected phonon modes of $\beta-\mathrm{Ga}_{2} \mathrm{O}_{3}$ in various crystal orientations for parallel polarized (black) and cross-polarized (red) configurations; experimental data (symbols) and fits (solid lines). The maximum intensity for each phonon mode is scaled to 1 . The vertical green and blue lines indicate the [102]- and [001]-direction, respectively

(532 nm, from [78]), these angular intensity patterns can be modeled consistently without additional parameters, allowing to determine the Raman tensor elements and their (relative) signs [128].

6 Summary and Outlook We have reviewed recent advances in the understanding of optically anisotropic crystals, namely the recently solved problem of modeling and interpreting Raman scattering intensities for excitation off a principal axis for arbitrary crystals, the dipole-based structure of the dielectric function due to lattice and electronic contributions and the dispersion of singular axes in the absorption regime. The transmission at an interface to a biaxial medium cannot be described with the current theory decomposing the wave in the biaxial medium into eigenstates.

Also particularities of microcavities with anisotropic cavity material, as composite systems from non-collinear uniaxial media, have been discussed. In such effectively biaxial system the complex energy dispersion has generally elliptically polarized eigenstates. We have predicted that modes in planar, anisotropic micro-cavities exhibit exceptional points (for suitable cavity thickness) and have demonstrated that in a (non-optimal) $\lambda$-cavity formed by a m-plane $(\mathrm{Mg}, \mathrm{Zn}) \mathrm{O} / \mathrm{ZnO}$ heterostructure the two modes are non-orthogonal and partially coalesced.

We foresee these insights to bear fruit in the understanding of complex materials and the characterization of thin films and the modeling of photonic devices from optically anisotropic materials.
In the light of the literature review compiled here, W. Voigt should be given credit for the first description and analysis of what is now called exceptional point.

Acknowledgements This work has been supported by Deutsche Forschungsgemeinschaft in the framework of Sonderforschungsbereich 762 (SFB 762) "Functionality of Oxide Interfaces" and within GR 1011/30-2 and by Universität Leipzig within research profile "Complex Matter".

\section{Appendix}

7.1 Formalism with Eigenstates in Biaxial Medium The problem of reflection and transmission of planar waves at the planar interface to a biaxial medium has been treated, e.g. in [129-134], and also for computer graphics/ray tracing $[135,136]$, however without paying special attention to the singular directions. We consider here a wave incoming from vacuum. The dielectric tensor is rotated to describe the orientation of the biaxial medium and the components of the rotated tensor shall be denoted by $\epsilon_{i j}$.

The wave vector of the incoming wave is $k=$ $(-\sin \alpha \sin \psi, \sin \alpha \cos \psi, \cos \alpha)$ (omitting the factor $k_{0}=\omega / c_{0}$, and working with $\left.|k|=1\right), \alpha$ being the angle of incidence relative to the interface normal $z, \psi$ being the azimuthal angle. The incoming wave shall be of type $s$ (v- or TM-type) or type $p=k \times s$ (h- or TE-type) polarization. The reflected wave has the wave vector $\boldsymbol{k}^{\prime}=\left(k_{x}, k_{y},-k_{z}\right)$ and the vectors $\boldsymbol{s}^{\prime}=\boldsymbol{s}$ and $\boldsymbol{p}^{\prime}=-\boldsymbol{k}^{\prime} \times s$, the transmitted wave has the wave vectors $\boldsymbol{k}_{\boldsymbol{a}}$ and $\boldsymbol{k}_{\boldsymbol{b}}$ of the eigenstates and the related electrical field vectors $\boldsymbol{a}$ and $\boldsymbol{b}$. 
One can follow the approach of [137] where the $z$ component of the $a$ - and $b$-waves in the biaxial medium is found from $|G|=0$ with

$$
G=\left(\begin{array}{cc}
-\left[b_{y x}+b_{z x} b_{y z} / c_{x y z}\right] & c_{x z y}-b_{z y} b_{y z} / c_{x y z} \\
-\left[c_{y z x}-b_{z x} b_{x z} / c_{x y z}\right] & b_{x y}+b_{x z} b_{z y} / c_{x y z}
\end{array}\right),
$$

and [138]

$$
\begin{aligned}
b_{i j} & =\epsilon_{i j}+k_{i} k_{j} \\
c_{i j k} & =k_{i}^{2}+k_{j}^{2}-\epsilon_{k k} .
\end{aligned}
$$

The four solutions of $|G|=0$ represent two backward and two forward waves, the latter are taken as those with positive real parts $k_{a, z}$ and $k_{b, z}$. For a given in-plane field $\left(E_{x}, E_{y}\right)$ the $z$-component of the electric field is then given by [137]

$$
E_{z}=\frac{b_{z x} E_{x}+b_{z y} E_{y}}{c_{x y z}} .
$$

The eigenvectors $\boldsymbol{a}$ and $\boldsymbol{b}$ are the (normalized) fields $\boldsymbol{E}_{\boldsymbol{a}}$ (using $k_{a, z}$ ) and $\boldsymbol{E}_{\boldsymbol{b}}$ (using $k_{b, z}$ ).

In the regions 1 (vacuum) and 2 (biaxial medium) the fields are

$\boldsymbol{E}_{\mathbf{1}}^{\boldsymbol{s}}=\boldsymbol{s} \exp \imath \boldsymbol{k} \boldsymbol{r}+\left(R_{s s} \boldsymbol{s}^{\prime}+R_{s p} \boldsymbol{p}^{\prime}\right) \exp \imath \boldsymbol{k}^{\prime} \boldsymbol{r}$

$\boldsymbol{E}_{\mathbf{2}}^{\boldsymbol{s}}=X_{s a} \boldsymbol{a} \exp \imath \boldsymbol{k}_{\boldsymbol{a}} \boldsymbol{r}+X_{s b} \boldsymbol{b} \exp \imath \boldsymbol{k}_{\boldsymbol{b}} \boldsymbol{r}$

$\boldsymbol{E}_{\mathbf{1}}^{\boldsymbol{p}}=\boldsymbol{p} \exp \imath \boldsymbol{k} \boldsymbol{r}+\left(R_{p s} \boldsymbol{s}^{\prime}+R_{p p} \boldsymbol{p}^{\prime}\right) \exp \imath \boldsymbol{k}^{\prime} \boldsymbol{r}$

$\boldsymbol{E}_{\mathbf{2}}^{\boldsymbol{p}}=X_{p a} \boldsymbol{a} \exp \imath \boldsymbol{k}_{\boldsymbol{a}} \boldsymbol{r}+X_{p b} \boldsymbol{b} \exp \imath \boldsymbol{k}_{\boldsymbol{b}} \boldsymbol{r}$.

The interface matching conditions for the $\boldsymbol{s}$ - and $\boldsymbol{p}$-fields read

$0=z \times\left(E_{1}^{\nu}-E_{2}^{\nu}\right)$

$0=\boldsymbol{z} \times\left[\nabla \times\left(\boldsymbol{E}_{\mathbf{1}}^{\boldsymbol{\nu}}-\boldsymbol{E}_{\mathbf{2}}^{\boldsymbol{\nu}}\right)\right]$,

with $\nu=\mathrm{s}$, p. The action of $\nabla \times$ on the exponentials is of the type $\imath \boldsymbol{k} \times$. The $x$ - and $y$-components of the two equations (14) and (15) provide 4 equations for the 4 variables $\left(R_{s s}, R_{s p}, X_{s a}, X_{s b}\right)$ and $\left(R_{p s}, R_{p p}, X_{p a}, X_{p b}\right)$, respectively.

Although put forward in [130,131] for non-absorbing crystals, this scheme has been used and elaborated in, e.g., Refs. [135, 137,139] also for absorbing crystals. We note that for this case it fails on and in the vicinity of the singular axes since the vectors $\boldsymbol{a}$ and $\boldsymbol{b}$ are no longer orthogonal (cmp. Fig. 7). Thus the transmitted wave cannot be properly projected and the transmission coefficients take unphysically large values $\gg 1$, eventually diverging on the singular axes.

7.2 Propagation-Formalism In order to avoid the problem of decomposing the field in the biaxial medium into eigenmodes we first follow the idea of the Berreman formalism [140, 141]. We start from the Maxwell equations (in the absence of currents and free charges) $\nabla \times \boldsymbol{E}=-\dot{\boldsymbol{B}}$ and $\nabla \times \boldsymbol{H}=\dot{\boldsymbol{D}}$ together with the special material equations $\boldsymbol{D}=\epsilon_{0} \boldsymbol{\epsilon} \boldsymbol{E}$ (The dielectric tensor $\boldsymbol{\epsilon}$ is given in the $(x, y, z)$ coordinates of the reflection geometry) and
$\boldsymbol{B}=\mu_{0} \boldsymbol{H}$ (nonmagnetic material). The periodicity in space and time of the fields shall be $\exp \imath\left(k_{x} x-\omega t\right)$. The curl operator is written as matrix $C$

$$
\boldsymbol{C}=\left(\begin{array}{ccc}
0 & -\partial_{z} & \partial_{y} \\
\partial_{z} & 0 & -\partial_{x} \\
-\partial_{y} & \partial_{x} & 0
\end{array}\right)=\left(\begin{array}{ccc}
0 & -\partial_{z} & 0 \\
\partial_{z} & 0 & -\imath k_{x} \\
0 & \imath k_{x} & 0
\end{array}\right)
$$

using the substitutions $\partial_{x}=\imath k_{x}$ and $\partial_{y}=0$. We set up equations for a six-component vector $\left(\boldsymbol{E}, \boldsymbol{H}^{\prime}\right)$ with $\boldsymbol{H}^{\prime}=$ $\mu_{0} c \boldsymbol{H}$, and arrive at (in $3 \times 3$ block matrix form)

$$
\left(\begin{array}{cc}
0 & C \\
-C & 0
\end{array}\right)\left(\begin{array}{c}
E \\
H^{\prime}
\end{array}\right)=\left(\begin{array}{cc}
\epsilon & 0 \\
0 & 1
\end{array}\right)\left(\begin{array}{c}
E \\
H^{\prime}
\end{array}\right) .
$$

The algebraic third and sixth row yield $E_{z}$ and $H_{z}^{\prime}$ in terms of the other field components. Eventually we find for the four-component vector $\boldsymbol{\Psi}$ of the tangential field components $\boldsymbol{E}_{\boldsymbol{T}}=\left(E_{x}, E_{y}\right)$ and $\boldsymbol{H}_{\boldsymbol{T}}^{\prime}=\left(H_{x}^{\prime}, H_{y}^{\prime}\right), \boldsymbol{\Psi}=$ $\left(E_{x}, E_{y}, H_{x}^{\prime}, H_{y}^{\prime}\right)$ :

$$
\frac{\partial}{\partial z} \boldsymbol{\Psi}=\imath k_{0} \boldsymbol{\Gamma} \boldsymbol{\Psi}
$$

with $k_{0}=\omega / c_{0}, k_{x}=k_{0} \sin \alpha, \alpha$ being the angle of incidence relative to the surface normal, and the matrix $\Gamma=$

$\left(\begin{array}{cccc}\frac{\epsilon_{z x} \sin \alpha}{\epsilon_{z z}} & \frac{\epsilon_{z y} \sin \alpha}{\epsilon_{z z}} & 0 & 1-\frac{\sin ^{2} \alpha}{\epsilon_{z z}} \\ 0 & 0 & -1 & 0 \\ \frac{\epsilon_{y z} \epsilon_{z x}}{\epsilon_{z z}}-\epsilon_{y x} & \sin ^{2} \alpha+\frac{\epsilon_{y z} \epsilon_{z y}}{\epsilon_{z z}}-\epsilon_{y y} & 0 & -\frac{\epsilon_{y z} \sin \alpha}{\epsilon_{z z}} \\ -\frac{\epsilon_{x z} \epsilon_{z x}}{\epsilon_{z z}}+\epsilon_{x x} & -\frac{\epsilon_{x z} \epsilon_{z y}}{\epsilon_{z z}}+\epsilon_{x y} & 0 & \frac{\epsilon_{x z} \sin \alpha}{\epsilon_{z z}}\end{array}\right)$,

similar to the result in [142]. Thus for a slab of homogeneous material and thickness $d$ the fields between entry $(z=0)$ and exit $(z=d)$ are related by

$$
\boldsymbol{\Psi}(0)=\exp \left(-\imath k_{0} d \boldsymbol{\Gamma}\right) \boldsymbol{\Psi}(d)=\boldsymbol{P} \boldsymbol{\Psi}(d) .
$$

The $4 \times 4$ propagation matrix $\boldsymbol{P}$ shall be written with $2 \times 2$ block matrices $\boldsymbol{P}_{i}$,

$$
\boldsymbol{P}=\left(\begin{array}{ll}
\boldsymbol{P}_{1} & \boldsymbol{P}_{2} \\
\boldsymbol{P}_{3} & \boldsymbol{P}_{4}
\end{array}\right)
$$

For the simulations executed in this paper the MatrixExp function of Mathematica [76] has been used for the evaluation of $\boldsymbol{P}$.

The (normalized) electromagnetic wave impinging from vacuum onto the surface has the wave vector $\boldsymbol{k}=k_{0}(\sin \alpha, 0, \cos \alpha)=k_{0} \hat{\boldsymbol{k}}$. The relation between the electrical and magnetic fields in vacuum are $\boldsymbol{H}^{i}=\hat{\boldsymbol{k}} \times \boldsymbol{E}^{i}$ and $\boldsymbol{E}^{i}=-\hat{\boldsymbol{k}} \times \boldsymbol{H}^{\prime i}$, from which follows the impedance matrix $Z$ (det $Z=1)$

$$
\boldsymbol{E}_{\boldsymbol{T}}{ }^{i}=\left(\begin{array}{cc}
0 & \cos \alpha \\
-\frac{1}{\cos \alpha} & 0
\end{array}\right)\left(\begin{array}{c}
H_{x}^{\prime i} \\
H_{y}^{\prime i}
\end{array}\right)=\boldsymbol{Z} \boldsymbol{H}_{\boldsymbol{T}}^{{ }^{i}},
$$


and at the top surface $(z=0)$ :

$$
\boldsymbol{E}_{\boldsymbol{T}}^{i}(0)=\boldsymbol{Z} \boldsymbol{H}_{\boldsymbol{T}}^{\boldsymbol{i}^{i}}(0),
$$

accordingly for the reflected and transmitted waves [143]

$$
\begin{aligned}
& \boldsymbol{E}_{\boldsymbol{T}}{ }^{r}(0)=-\boldsymbol{Z} \boldsymbol{H}_{\boldsymbol{T}}^{{ }^{r}}(0) \\
& \boldsymbol{E}_{\boldsymbol{T}}{ }^{t}(d)=\boldsymbol{Z} \boldsymbol{H}_{\boldsymbol{T}}^{\prime{ }^{t}}(d) .
\end{aligned}
$$

We note that the theory is valid for all angles of incidence $\alpha$; in this paper only normal incidence is considered, obviously then

$$
\boldsymbol{Z}=\left(\begin{array}{cc}
0 & 1 \\
-1 & 0
\end{array}\right)
$$

Reflection and transmission are described by the matrices $\boldsymbol{R}$ and $\boldsymbol{T}$,

$$
\begin{aligned}
& \boldsymbol{E}_{\boldsymbol{T}}^{r}(0)=\boldsymbol{R} \boldsymbol{E}_{\boldsymbol{T}}{ }^{i}(0) \\
& \boldsymbol{E}_{\boldsymbol{T}}{ }^{t}(d)=\boldsymbol{T} \boldsymbol{E}_{\boldsymbol{T}}{ }^{i}(0),
\end{aligned}
$$

and from (20) eventually it is found [143]

$$
\begin{gathered}
\boldsymbol{R}=\left[\boldsymbol{P}_{1} \boldsymbol{Z}+\boldsymbol{P}_{2}-\boldsymbol{Z}\left(\boldsymbol{P}_{3} \boldsymbol{Z}+\boldsymbol{P}_{4}\right)\right] \\
{\left[\boldsymbol{P}_{1} \boldsymbol{Z}+\boldsymbol{P}_{2}+\boldsymbol{Z}\left(\boldsymbol{P}_{3} \boldsymbol{Z}+\boldsymbol{P}_{4}\right)\right]^{-1}} \\
\boldsymbol{T}=2 \boldsymbol{Z}\left[\boldsymbol{P}_{1} \boldsymbol{Z}+\boldsymbol{P}_{2}+\boldsymbol{Z}\left(\boldsymbol{P}_{3} \boldsymbol{Z}+\boldsymbol{P}_{4}\right)\right]^{-1} .
\end{gathered}
$$

With varying slab thickness $d$, the transmission and reflection exhibit thickness (Fabry-Pérot type) oscillations. In the case of absorption these die out if the thickness is large compared to the absorption length. Then the reflectivity value for a thick layer can be obtained. The transmitted intensity contains the mode conversion during the propagation in the slab.

\section{References}

[1] As is always the case with symmetry and group theoretical classifications, accidental degeneracies, here with respect to the values of the index of refraction in different directions, can occur.

[2] Woldemar Voigt (1850-1919) [3-6] was born in Leipzig, was pupil at the Thomasschule and studied mathematics and physics at Universität Leipzig. He continued his studies at Königsberg and received his doctoral degree. After his habilitation in Leipzig he became außerordentlicher professor in Königsberg. In Königsberg he was trained by F. Neumann who had awakened his interest in the physics of crystals, beginning with their elastic properties (PhD thesis on "Untersuchung der Elasticitätsverhältnisse des Steinsalzes"). 1883 he became professor in Göttingen where he stayed until his death. He developed an early version of the Lorentz transformation and contributed significantly to the field of crystals, in particular piezoelectricity and crystal optics, and the understanding of the Zeeman effect. For the $\mathrm{ZnO}$ community it may be interesting that Voigt is the grandfather of E. Mollwo [7] (son of Erika Maria Voigt).

[3] C. Runge, Woldemar Voigt (1850-1919), Physikal. Zeitschrift 21, 81-82 (1920).
[4] K. Försterling, Woldemar Voigt zum 100. Geburtstage, Die Naturwissenschaften 38, 217-221 (1951).

[5] M. Päsler, Einiges aus der Geschichte der Kristallkunde. Zur 50sten Wiederkehr des Todestages von Woldemar Voigt, Physikalische Blätter 25, 542-546 (1969).

[6] D. Hoffmann, Paul Drude (1863-1906) Ann. Phys. (Leipzig) 15, 449-460 (2006).

[7] E. Mollwo, Zur Vor- und Frühgeschichte der Festkörperphysik in Deutschland, Wege in die Physikdidaktik, Band 3 (Verlag Palm \& Enke, Erlangen, 1993), pp. 1-10.

[8] W. Voigt, Beiträge zur Aufklärung der Eigenschaften pleochroitischer Kristalle, Ann. Phys. 314, 367-416 (1902).

[9] W. Voigt, Beiträge zur Aufklärung der Eigenschaften pleochroitischer Krystalle Nachrichten von der Gesellschaft der Wissenschaften zu Göttingen, Mathematisch-Physikalische Klasse 1902, pp. 48-91.

[10] W. Voigt, Weiteres zur Aufklärung der Eigenschaften pleochroitischer Krystalle Nachrichten von der Gesellschaft der Wissenschaften zu Göttingen, Mathematisch-Physikalische Klasse 1902, pp. 269-277.

[11] A. Akbarzadeh, C.-W. Qiu, A.J. Danner, Exploiting design freedom in biaxial dielectrics to enable spatially overlapping optical instruments, Sci. Rep. 3, 2055 (2013).

[12] W.R. Hamilton, Third supplement to an essay on the theory of systems of rays, Trans. R. Irish Acad. 17, 1-144 (1837).

[13] H. Lloyd, On the phenomena presented by light in its passage along the axes of biaxial crystals, Phil. Mag. 1, 112120 and 207-210 (1833).

[14] M.V. Berry, M.R. Jeffrey, J.G. Lunney, Conical diffraction: observations and theory, Proc. R. Soc. A 462, 1629-1642 (2006).

[15] D.P. O'Dwyer, C.F. Phelan, Y.P. Rakovich, P.R. Eastham, J.G. Lunney, J.F. Donegan, Generation of continuously tunable fractional optical orbital angular momentum using internal conical diffraction, Optics Express 18, 16480-16485 (2010).

[16] H. Laspeyres, Mineralogische Bemerkungen (VII. Theil), Z. Kristallographie und Mineralogie 4, 433-467 (1880).

[17] "Es ist deshalb möglich, dass in monoklinen Krystallen diese beiden innerhalb der Symmetrieebene liegenden 'Absorptionsaxen' in ihrer Richtung unabhängig von der Lage der daselbst befindlichen Elasticitätsaxen sind. [...] In triklinen Krystallen ist bekanntlich die Lage der drei zu einander normalen Elasticitätsaxen ganz unabhängig von der Krystallform, es können deshalb theoretisch die drei 'Absorptionsaxen' in ihrer Lage ebenfalls ganz unabhängig nicht nur von der Krystallform, sondern auch von der Lage der Elasticitätsaxen sein.", from [16]. It is thus possible that in monoclinic crystals the directions of the two 'absorption axes' lying within the symmetry plane are independent from the elasticity axes. [...] In triclinc crystals it is known that the three orthogonal elasticity axes are independent of the crystal shape, thus theoretically the orientation of the three 'absorption axes' can be completely independent not only from the crystal shape but also from the orientation of the elasticity axes.

[18] M. Babinet, Sur l'absorption dans les milieux colores birefringents, Comptes Rendus 7, 832-833 (1838).

[19] W. Voigt, Zur Theorie der Absorption des Lichtes in Krystallen, Ann. Phys. 259, 577-606 (1884). 
[20] H. Becquerel, Sur I'absorption de la lumiere au travers des cristaux, Bull. Société Français de Minéralogie 10, 120-124 (1887).

[21] P. Drude, Ueber die Gesetze der Reflexion und Brechung des Lichtes an der Grenze absorbirender Krystalle, Ann. Phys. 268, 584-625 (1887).

[22] "Optische Axen gibt es also in einem absorbirenden Krystalle rhombischen Systems nicht.", from [21]. Optic axes thus do not exist in an absorbing crystal of the rhombic system.

[23] R. Brauns, Die optischen Anomalien der Krystalle, Preisschriften der Fürstlich Jablonowski'schen Gesellschaft zu Leipzig XXIX (S. Hirzel, Leipzig, 1891), pp. 1-370.

[24] Deutsches Museum Archiv, München.

[25] W. Voigt, Theoretisches und Experimentelles zur Aufklärung des optischen Verhaltens aktiver Kristalle, Ann. Physik 323, 645-694 (1905).

[26] W. Voigt, Bemerkungen zur Theorie der konischen Refraktion, Ann. Physik 324, 14-21 (1906).

[27] W. Voigt, Über die sogenannte innere konische Refraktion bei pleochroitischen Kristallen, Ann. Physik 325, 108-126 (1906).

[28] W. Voigt, Über singuläre Richtungen in pleochroitischen Kristallen, Ann. Physik 332, 1002-1022 (1908).

[29] W. Voigt, Ein optisches Paradoxon, Ann. Physik 332, 10231024 (1908).

[30] M. Berek, Die singulären optischen Richtungen (Windungsachsen) in beliebig stark absorbierenden Kristallen rhombischer Symmetrie, Z. Kristallographie 80, 18-36 (1931).

[31] M. Berek, Elementare Einführung in die Optik absorbierender Kristalle und in die Methodik ihrer Bestimmung im reflektierten Licht, Centralblatt für Mineralogie, Geologie und Palaeontologie, Abt. A (1931), pp. 198-209.

[32] S. Pancharatnam, The propagation of light in absorbing biaxial crystals - I. Theoretical, Proc. Indian Acad. Sci A 42 , 86-109 (1955).

[33] S. Pancharatnam, The propagation of light in absorbing biaxial crystals - II. Experimental, Proc. Indian Acad. Sci A 42, 235-248 (1955).

[34] A.P. Khapalyuk, On the theory of circular optical axes, Opt. Spectrosc. (USSR), 12, 52-54 (1962).

[35] B.N. Grechushnikov, A.F. Konstantinova, Crystal optics of absorbing and gyrotropic media, Comput. Math. Applic. 16, 637-655 (1988).

[36] J. Gerardin, A. Lakhtakia, Conditions for Voigt wave propagation in linear, homogeneous, dielectric mediums, Optik 112, 493-495 (2001).

[37] T.G. Mackay, A. Lakhtatia, Voigt wave propagation in biaxial composite materials, J. Opt. A: Pure Appl. Opt. 5, 91-95 (2003).

[38] M.V. Berry, M.R. Dennis, The optical singularities of birefringent dichroic chiral crystals, Proc. R. Soc. London A 459, 1261-1292 (2003).

[39] S. Joly, Y. Petit, B. Boulanger, P. Segonds, C. Félix, B. Ménaert, G. Aka, Singular topology of optical absorption in biaxial crystals, Opt. Express 17, 19868-19873 (2009).

[40] V.S. Merkulov, Cones of Singular Optic Axes in Monoclinic and Triclinic Crystals, Optics and Spectroscopy 114, 563567 (2013).
[41] T.G. Golovina, A.F. Konstantinova, K.K. Konstantinov, E.A. Evdishchenko, Number of optic axes in Absorbing Triclinic Crystals, Crystallography Reports 58, 877-882 (2013).

[42] C. Sturm, M. Grundmann, The singular axes in Biaxial Crystals and Analysis of Their Spectral Dispersion Effects in $\beta-\mathrm{Ga}_{2} \mathrm{O}_{3}$, Phys. Rev. A 93, 053839 (2016).

[43] A.N. Grigorenko, Quantum mechanics with a nonHermitian Hamiltonian, Physics Lett. A 172, 350-354 (1993).

[44] C.M. Bender, S. Boettcher, Real Spectra in Non-Hermitian Hamiltonians Having $\mathcal{P} \mathcal{T}$ Symmetry, Phys. Rev. Lett. 80, 5243-5246 (1998).

[45] C.M. Bender, Making sense of non-Hermitian Hamiltonians, Rep. Prog. Phys. 70, 947-1018 (2007).

[46] Special Issue: Quantum Physics with non-Hermitian Operators: Theory and Experiment, Fortschr. Phys. 61, 51-419 (2013).

[47] W.D. Heiss, H.L. Harney, The chirality of exceptional points, Eur. Phys. J. D - Atomic, Molecular, Optical and Plasma Physics 17, 149-151 (2001).

[48] F. Keck, H.J. Korsch, S. Mossmann, Unfolding a diabolic point: a generalized crossing scenario, J. Phys. A: Math. Gen. 36, 2125-2137 (2003).

[49] W.D. Heiss, Exceptional points of non-Hermitian operators, J. Phys. A: Math. Gen. 37, 2455-2464 (2004).

[50] M.V. Berry, Physics of nonhermitian degeneracies, Czech. J. Phys. 54, 1039-1047 (2004).

[51] B. Zhen, C.W. Hsu, Y. Igarashi, L. Lu, I. Kaminer, A. Pick, S.-L. Chua, J.D. Joannopoulos, M. Soljačcić, Spawning rings of exceptional points out of Dirac cones, Nature 525, 354-358 (2015).

[52] H. Eleuch, I. Rotter, Clustering of exceptional points and dynamical phase transitions, Phys. Rev. A 93, 042116 (2016)

[53] J.-W. Ryu, W.-S. Son, D.-U. Hwang, S.-Y. Lee, S.W. Kim, Exceptional points in coupled dissipative dynamical systems, Phys. Rev. E 91, 052910 (2015)

[54] O.N. Kirillov, Exceptional and diabolical points in stability questions, Fortschr. Phys. 61, 205-224 (2013).

[55] T. Stehmann, W.D. Heiss, F.G. Scholtz, Observation of exceptional points in electronic circuits, J. Physics A: Mathematical and General 37, 7813-7819 (2004)

[56] C. Dembowski, H.-D. Gräf, H.L. Harney, A. Heine, W.D. Heiss, H. Rehfeld, A. Richter, Experimental Observation of the Topological Structure of Exceptional Points, Phys. Rev. Lett. 86, 787-790 (2001).

[57] M. Kammerer, F. Merz, F. Jenko, Exceptional points in linear gyrokinetics, Phys. Plasmas 15, 052102 (2016)

[58] D.D. Smith, N.N. Lepeshkin, A. Schweinsberg, G. Gehring, R.W. Boyd, Q-H. Park, H. Chang, D.J. Jackson, Coupledresonator-induced transparency in a fiber system, Opt. Comm. 264, 163-168 (2006).

[59] H. Cartarius, J. Main, G. Wunner, Exceptional Points in Atomic Spectra, Phys. Rev. Lett. 99, 173003 (2007).

[60] H. Menke, M. Klett, H. Cartarius, J. Main, G. Wunner, State flip at exceptional points in atomic spectra, Phys. Rev. A 93, 013401 (2016)

[61] M. Brandstetter, M. Liertzer, C. Deutsch, P. Klang, J. Schöberl, H.E. Türeci, G. Strasser, K. Unterrainer, S. Rotter, Reversing the pump dependence of a laser at an exceptional point, Nat. Comm. 5, 4034 (2014). 
[62] Z. Lin, A. Pick, M. Lončar, A.W. Rodriguez, Enhanced Spontaneous Emission at Third-Order Dirac Exceptional Points in Inverse-Designed Photonic Crystals, Phys. Rev. Lett. 117, 107402 (2016)

[63] G. Yoo, H.-S. Sim, H. Schomerus, Quantum noise and mode nonorthogonality in non-Hermitian $\mathcal{P} \mathcal{T}$-symmetric optical resonators, Phys. Rev. A 84, 063833 (2011).

[64] J.-H. Wu, M. Artoni, G.C. La Rocca, Non-Hermitian Degeneracies and Unidirectional Reflectionless Atomic Lattices, Phys. Rev. Lett. 113, 123004 (2014).

[65] Y. Huang, G. Veronis, C. Min, Unidirectional reflectionless propagation in plasmonic waveguide-cavity systems at exceptional points, Opt. Express 23, 29882-29895 (2015).

[66] J.-W. Ryu, S.-Y. Lee, S.W. Kim, Analysis of multiple exceptional points related to three interacting eigenmodes in a non-Hermitian Hamiltonian, Phys. Rev. A 85, 042101 (2012).

[67] D.I. Borisov, F. Ružička, M. Znojil, Multiply Degenerate Exceptional Points and Quantum Phase Transitions, Int. J. Theor. Phys. 54, 4293-4305 (2015).

[68] K. Ding, G. Ma, M. Xiao, Z.Q. Zhang, C.T. Chan, Emergence, Coalescence, and Topological Properties of Multiple Exceptional Points and Their Experimental Realization, Phys. Rev. X 6, 021007 (2016).

[69] H. Michalke, Quantitative Untersuchungen der Polarisation der Raman-Linien von Kristallen, Z. Physik 108, 748-770 (1938).

[70] H.C. Lin, Z.C. Feng, M.S. Chen, Z.X. Shen, I.T. Ferguson, W. Lu, Raman scattering study on anisotropic property of wurtzite GaN, J. Appl. Phys. 105, 036102 (2009).

[71] T. Sander, S. Eisermann, B.K. Meyer, P.J. Klar, Raman tensor elements of wurtzite ZnO, Phys. Rev. B 85, 165208 (2012).

[72] J. Kim, J.-U. Lee, J. Lee, H.J. Park, Z. Lee, C. Lee, H. Cheong, Anomalous polarization dependence of Raman scattering and crystallographic orientation of black phosphorus, Nanoscale 7, 18708-18715 (2015).

[73] M. Suzuki, T. Yokoyama, M. Ito, Polarized Raman spectra of naphthalene and anthracene single crystals, Spectrochimica Acta Part A: Molecular Spectroscopy 24, 10911107 (1968)

[74] C. Kranert, C. Sturm, R. Schmidt-Grund, M. Grundmann, Raman Tensor Formalism for Optically Anisotropic Crystals, Phys. Rev. Lett. 116, 127401 (2016).

[75] M. Grundmann, J. Zúñiga-Pérez, Pseudomorphic ZnObased heterostructures: from polar through all semipolar to nonpolar orientations, phys. stat. sol. (b) 253, 351-360 (2016).

[76] Computer code Mathematica, Version 10.0.2, Wolfram Research, Champaign, IL, 2014.

[77] Computer code MATLAB, Version R2008b, MathWorks, Natick, MA, 2015.

[78] C. Sturm, J. Furthmüller, F. Bechstedt, R. Schmidt-Grund, M. Grundmann, Dielectric tensor of monoclinic $\mathrm{Ga}_{2} \mathrm{O}_{3} \mathrm{sin}$ gle crystals in the spectral range 0.5-8.5 eV, APL Mater. 3, 106106 (2015).

[79] Tamura Corporation, www.tamura-ss.co.jp/en/gao/

[80] C. Sturm, R. Schmidt-Grund, C. Kranert, J. Furthmüller, F. Bechstedt, M. Grundmann, Dipole Analysis of the Dielectric Function of Color Dispersive Materials: Application to Monoclinic $\mathrm{Ga}_{2} \mathrm{O}_{3}$, Phys. Rev. B 94, 035148 (2016).
[81] M. Schubert, R. Korlacki, S. Knight, T. Hofmann, S. Schöche, V. Darakchieva, E. Janzén, B. Monemar, D. Gogova, Q.-T. Thieu, R. Togashi, H. Murakami, Y. Kumagai, K. Goto, A. Kuramata, S. Yamakoshi, M. Higashiwaki, Anisotropy, phonon modes, and free charge carrier parameters in monoclinic $\beta$-gallium oxide single crystals, Phys. Rev. B 93, 125209 (2016).

[82] T.G. Mayerhöfer, S. Weber, J. Popp, Dispersion analysis of perpendicular modes in anisotropic crystals and layers, J. Opt. Soc. Am. A 28, 2428-2435 (2011).

[83] T.G. Mayerhöfer, V. Ivanovski, J. Popp, Dispersion analysis of non-normal reflection spectra from monoclinic crystals, Vib. Spectrosc. 63, 396-03 (2012).

[84] M. Born, Dynamik der Kristallgitter (B.G. Teubner, Leipzig, 1915); M. Born, K. Huang, Dynamical Theory of Crystal Lattices (Clarendon Press, Oxford, 1954).

[85] R. Claus, The Lattice Dynamical Background of Classical Crystal Optics, phys. stat. sol. (b) 100, 9-41 (1980).

[86] A.G. Emslie, J.R. Aronson, Determination of the complex dielectric tensor of triclinic crystals: theory, J. Opt. Soc. Am. 73, 916-919 (1983).

[87] M. Schubert, The Lyddane-Sachs-Teller relationship for polar vibrations in materials with monoclinic and triclinic crystal systems, arXiv: 1602.08785.

[88] M. Grundmann, T. Böntgen, M. Lorenz, The Occurrence of Rotation Domains in Heteroepitaxy, Phys. Rev. Lett. 105, 146102 (2010).

[89] M. Grundmann, Formation of Epitaxial Domains: Unified Theory and Survey of Experimental Results, phys. stat. sol. (b) 248, 805-824 (2011).

[90] T. Oshima, T. Okuno, S. Fujita, $\mathrm{Ga}_{2} \mathrm{O}_{3}$ Thin Film Growth on c-Plane Sapphire Substrates by Molecular Beam Epitaxy for Deep-Ultraviolet Photodetectors, Jpn. J. Appl. Phys. 46, 7217-7220 (2007).

[91] R. Schmidt-Grund, C. Kranert, T. Böntgen, H. von Wenckstern, H. Krauß, M. Grundmann, Dielectric function in the NIR-VUV spectral range of $\left(\operatorname{In}_{x} G a_{1-x}\right)_{2} \mathrm{O}_{3}$ thin films, J. Appl. Phys. 116, 053510 (2014).

[92] N.M. Lawandy, S.A. Johnston, J. Martorell, Birefringence and polarization dependent absorption in sheared colloidal crystals, Opt. Comm. 65, 425-429 (1988).

[93] M. Sabooni, A.N. Nilsson, G. Kristensson, L. Rippe, Wave propagation in birefringent materials with off-axis absorption or gain, Phys. Rev. A 93, 013842 (2016).

[94] J.M. Virgós Rovira, S.A. Abrego, V.H. Rodríguez, C.M. Pascual, Refractive indices and absorption coefficients associated with any propagation direction of a wave in $a b$ sorbing biaxial materials, J. Optics 19, 187-192 (1988).

[95] C. Alberdi, J.M. Diñeiro, B. Hernández, C. Sáenz, General expressions for the refractive indices of absorbing biaxial media as a function of the angle of incidence, J. Opt. Soc. Am. A 32, 228-237 (2015).

[96] R. Cattoor, I Manek-Hönninger, M. Tondusson, Ph. Veber, T.K. Kalkandjiev, D. Rytz, L. Canioni, M. Eichhorn, Wavelength dependence of the orientation of optic axes in $K G W$, Applied Physics B - Laser and Optic 116, 831-836 (2014).

[97] The components of the dielectric tensor of $\beta-\mathrm{Ga}_{2} \mathrm{O}_{3}$ at $E=$ $6.20 \mathrm{eV}$ are (taken from [78]):

$\epsilon_{x x}=4.366+\imath 1.592, \epsilon_{y y}=5.016+\imath 2.150$,

$\epsilon_{z z}=4.686+\imath 1.460, \epsilon_{x z}=0.094+\imath 0.017$. 
[98] J.F. Nye, Lines of Circular Polarization in Electromagnetic Wave Fields, Proc. Roy. Soc. A 389, 279-290 (1983).

[99] M.R. Dennis, Polarization singularities in paraxial vector fields: morphology and statistics, Opt. Comm. 213, 201-221 (2002).

[100] F. Flossmann, U.T. Schwarz, M. Maier, M.R. Dennis, Polarization Singularities from Unfolding an Optical Vortex through a Birefringent Crystal, Phys. Rev. Lett. 95, 253901 (2005).

[101] S.-Y. Lu, R.A. Chipman, Homogeneous and inhomogeneous Jones matrices, J. Opt. Soc. Am. A 11 766-773 (1994).

[102] P. Drude, Das Verhalten der Absorptionsco efficienten Krystallen, Ann. Physik 276, 665-680 (1890).

[103] "a surface with two shells [... that] are connect along certain arcs that lie asymmetric with respect to the coordinate planes for monoclinic and triclinic crystals."

[104] W.D. Heiss, Repulsion of resonance states and exceptional points, Phys. Rev. E 61, 929-932 (2000).

[105] R.M. Denning, J.A. Mandarino, Pleochroism in synthetic ruby, American Mineralogist 40, 1055-1061 (1955).

[106] C.V. Raman, The birefringence patterns of crystal spheres, Proc. Indian Acad. Sci. A 43, 1-3 (1956).

[107] P. Enders, A. Bärwolff, M. Woerner, D. Suisky, $\boldsymbol{k} \cdot \boldsymbol{p}$ theory of energy bands, wave functions, and optical selection rules in strained tetrahedral semiconductors, Phys. Rev. B 51, 16695-16704 (1995).

[108] S. Klaiman, U. Günther, N. Moiseyev, Visualization of Branch Points in PT-Symmetric Waveguides, Phys. Rev. Lett. 101, 080402 (2008).

[109] S. Pancharatnam, Light propagation in absorbing crystals possessing optical activity - Electromagnetic theory, Proc. Indian Acad. Sci. A 48, 227-244 (1958).

[110] A. Brenier, Voigt wave investigation in the $\mathrm{KGd}\left(\mathrm{WO}_{4}\right)_{2}: \mathrm{Nd}$ biaxial laser crystal, J. Optics 17, 075603 (2015).

[111] C. Sturm, H. Hilmer, B. Rheinländer, R. SchmidtGrund, M. Grundmann, Cavity-photon dispersion in onedimensional confined microresonators with an optically anisotropic cavity material, Phys. Rev. B 83, 205301 (2011).

[112] T. Zhu, A. Dussaigne, G. Christmann, C. Pinquier, E. Feltin, D. Martin, R. Butté, N. Grandjean, Nonpolar GaNbased microcavity using AlN/GaN distributed Bragg reflector, Appl. Phys. Lett. 92, 061114 (2008).

[113] G. Rossbach, J. Levrat, A. Dussaigne, G. Cosendey, M. Glauser, M. Cobet, R. Butté, N. Grandjean, H. Teisseyre, M. Bockowski, I. Grzegory, T. Suski, Tailoring the light-matter coupling in anisotropic microcavities: Redistribution of oscillator strength in strained m-plane GaN/AlGaN quantum wells, Phys. Rev. B 84, 115315 (2011).

[114] R. Tao, M. Arita, S. Kako, Y. Arakawa, Fabrication and optical properties of non-polar III-nitride air-gap distributed Bragg reflector microcavities, Appl. Phys. Lett. 103, 201118 (2013).

[115] J. Zúñiga-Pérez, L. Kappei, C. Deparis, F. Reveret, M. Grundmann, E. de Prado, O. Jamadi, J. Leymarie, S. Chenot, M. Leroux, Homoepitaxial nonpolar (1010) ZnO/ZnMgO heterostructures: from single layers to monolithic Bragg reflectors and optical microcavities, Appl. Phys. Lett. 108, 251904 (2016).
[116] S. Richter, T. Michalsky, L. Fricke, C. Sturm, H. Franke, M. Grundmann, R. Schmidt-Grund, Maxwell consideration of polaritonic quasi-particle Hamiltonians in multi-level systems, Appl. Phys. Lett. 107, 231104 (2015).

[117] S. Richter, T. Michalsky, C. Sturm, B. Rosenow, M. Grundmann, R. Schmidt-Grund, Exceptional points in anisotropic planar microcavities, arXiv:1609.07653 (2016).

[118] J. Wiersig, Sensors operating at exceptional points: General theory, Phys. Rev. A 93, 033809 (2016).

[119] B. Peng, Ş.K. Özdemir, M. Liertzer, W. Chen, J. Kramer, H. Yilmaz, J. Wiersig, S. Rotter, L. Yang, Chiral modes and directional lasing at exceptional points, PNAS 113, 68456850 (2016).

[120] S.P.S. Porto, J.A. Giordmaine, T.C. Damen, Depolarization of Raman Scattering in Calcite, Phys. Rev. 147, 608-611 (1966).

[121] I.R. Beattie, T.R. Gilson, Single Crystal Laser Raman Spectroscopy, Proc. R. Soc. A 307, 407-429 (1968).

[122] P. Dawson, Polarisation measurements in Raman spectroscopy, Spectrochimica Acta Part A: Molecular Spectroscopy 28, 715-723 (1972).

[123] A. Rulmont, J.P. Flamme, Birefringence effect in the Raman spectrum of a crystal which is not cut parallel to the principal axes - I., Spectrochimica Acta Part A: Molecular Spectroscopy 35, 629-633 (1979).

[124] A. Rulmont, J.P. Flamme, M.J. Pottier, B.M. Wanklyn, Birefringence effect in the Raman spectrum of a crystal which is not cut parallel to the pricipal axes - II. Application to a single crystal of $\mathrm{LaBO}_{3}$, Spectrochimica Acta Part A: Molecular Spectroscopy 35, 635-639 (1979).

[125] N. Mao, J. Wu, B. Han, J. Lin, L. Tong, J. Zhang, Birefringence-Directed Raman Selection Rules in 2D Black Phosphorus Crystals, Small 12, 2627-2633 (2016).

[126] G. Pezzotti, H. Sueoka, A.A. Porporati, M.Manghnani, W. Zhu, Raman tensor elements for wurtzitic GaN and their application to assess crystallographic orientation at film/substrate interfaces, J. Appl. Phys. 110, 013527 (2011).

[127] D. Dohy, G. Lucazeau, A. Revcolevschi, Raman spectra and valence force field of single-crystalline $\beta-\mathrm{Ga}_{2} \mathrm{O}_{3}, \mathrm{~J}$. Solid State Chem. 45, 180-192 (1982).

[128] C. Kranert, C. Sturm, R. Schmidt-Grund, M. Grundmann, Raman tensor elements of $\beta-G a_{2} \mathrm{O}_{3}$, Sci. Rep. (2016), accepted; arXiv:1606.07409 (2016).

[129] S. Mudaliar, Green's functions for an anisotropic medium: Part I. Unbound case, Interim Report, RL-TR-93-264 (Rome Laboratory, Griffiss AFB, NY, 1993).

[130] S. Mudaliar, Green's functions for an anisotropic medium: Part II. Two-layer case, Interim Report, RL-TR-93-265 (Rome Laboratory, Griffiss AFB, NY, 1993).

[131] G.D. Landry, T.A. Maldonado, Complete method to determine transmission and reflection characteristics at a planar interface between arbitrarily oriented biaxial media, J. Opt. Soc. Am. A 12, 2048-2063 (1995).

[132] S. Mudaliar, J.K. Lee, Dyadic Green's functions for a two-layer biaxially anisotropic medium, J. Electromagnetic Waves and Applications 10, 909-923 (1996).

[133] G.F. Pettis, Hertzian dipoles and microstrip circuits on arbitrarily oriented biaxially anisotropic media, $\mathrm{PhD}$ Thesis, Rochester Institute of Technology (2008). 
[134] Y. Huang, J.K. Lee, Dyadic Green's functions for unbounded and two-layered general anisotropic media, Prog. Electromagnetics Research 30, 27-46 (2011).

[135] Y. Wang, P. Shi, H. Xin, L. Wu, Complex ray tracing in biaxial anisotropic absorbing media, J. Opt. A: Pure Appl. Opt. 10, 075009 (2008).

[136] P. Latorre, F.J. Seron, D. Gutierrez, Birefringence: calculation of refracted ray paths in biaxial crystals, Vis. Comput. 28, 341-356 (2012).

[137] I. Abdulhalim, Exact $2 \times 2$ matrix method for the transmission and reflection at the interface between two arbitrarily oriented biaxial crystals, J. Opt. A: Pure Appl. Opt. 1, 655661 (1999).

[138] We have simplified the notation and note that in equation (10b) of [137] is a misprint.

[139] J.W. Graham, J.K. Lee, Reflection and transmission from biaxially anisotropic-isotropic interfaces, Progress in Electromagnetics Research 136, 681-702 (2013).

[140] D.W. Berreman, Optics in stratified and anisotropic media: $4 \times 4$ matrix formulation, J. Opt. Soc. Am. 62, 502-510 (1972).

[141] M. Schubert, Polarization-dependent optical parameters of arbitrarily anisotropic homogeneous layered systems, Phys. Rev. B 53, 4265-4274 (1996).

[142] S. Stallinga, Berreman $4 \times 4$ matrix method for reflective liquid crystal displays, J. Appl. Phys. 85, 3023-3031 (1999).

[143] D. Zarifi, H. Oraizi, M. Soleimani, A. Abdolali, Analysis of Reflection and Transmission from Biaxial Chiral Slabs Using the State Space Approach, 2013 13th Mediterranean Microwave Symposium (MMS) (IEEE, Piscataway, 2013). 